\title{
Bifurcations of mixed-mode oscillations in three-timescale systems: An extended prototypical example
}

\author{
Kaklamanos, Panagiotis; Popovi, N. ; Kristiansen, Kristian Uldall
}

Published in:

Chaos

Link to article, DOI:

$10.1063 / 5.0073353$

Publication date:

2022

Document Version

Peer reviewed version

Link back to DTU Orbit

Citation $(A P A)$ :

Kaklamanos, P., Popovi, N., \& Kristiansen, K. U. (2022). Bifurcations of mixed-mode oscillations in threetimescale systems: An extended prototypical example. Chaos, 32, [013108 ]. https://doi.org/10.1063/5.0073353

\section{General rights}

Copyright and moral rights for the publications made accessible in the public portal are retained by the authors and/or other copyright owners and it is a condition of accessing publications that users recognise and abide by the legal requirements associated with these rights.

- Users may download and print one copy of any publication from the public portal for the purpose of private study or research.

- You may not further distribute the material or use it for any profit-making activity or commercial gain

- You may freely distribute the URL identifying the publication in the public portal

If you believe that this document breaches copyright please contact us providing details, and we will remove access to the work immediately and investigate your claim 


\section{Bifurcations of mixed-mode oscillations in three-timescale systems: An extended prototypical example ${ }^{\oplus}$}

Cite as: Chaos 32, 013108 (2022); https://doi.org/10.1063/5.0073353

Submitted: 29 September 2021 • Accepted: 20 December 2021 • Published Online: 11 January 2022

(D) P. Kaklamanos, (D) N. Popović and K. U. Kristiansen

\section{COLLECTIONS}

EP This paper was selected as an Editor's Pick
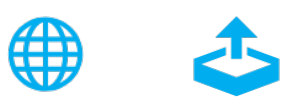

\section{ARTICLES YOU MAY BE INTERESTED IN}

Learning continuous chaotic attractors with a reservoir computer

Chaos: An Interdisciplinary Journal of Nonlinear Science 32, 011101 (2022); https://

doi.org/10.1063/5.0075572

Learn bifurcations of nonlinear parametric systems via equation-driven neural networks

Chaos: An Interdisciplinary Journal of Nonlinear Science 32, 011102 (2022); https://

doi.org/10.1063/5.0078306

The transition to synchronization on branching hierarchical lattices

Chaos: An Interdisciplinary Journal of Nonlinear Science 32, 013120 (2022); https://

doi.org/10.1063/5.0055291

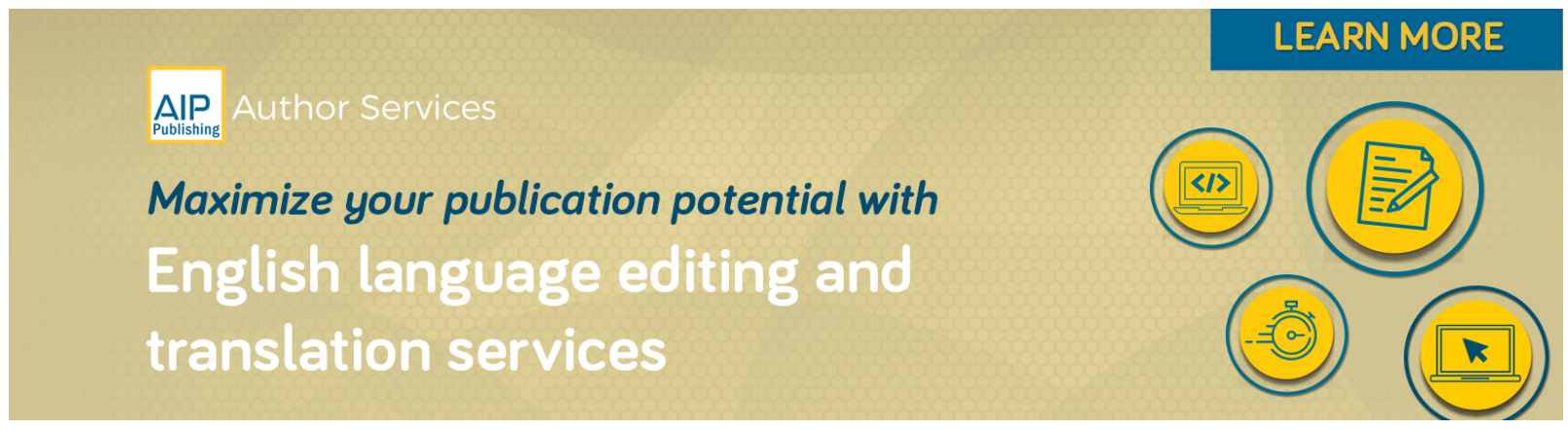




\title{
Bifurcations of mixed-mode oscillations in three-timescale systems: An extended prototypical example
}

\author{
Cite as: Chaos 32, 013108 (2022); doi: 10.1063/5.0073353 \\ Submitted: 29 September 2021 . Accepted: 20 December 2021 . \\ Published Online: 11 January 2022
}

P. Kaklamanos, , ,a) (D) N. Popović, , ,b) (D) and K. U. Kristiansen ${ }^{2, c)}$

\begin{abstract}
AFFILIATIONS
${ }^{1}$ School of Mathematics and Maxwell Institute for Mathematical Sciences, University of Edinburgh, James Clerk Maxwell Building, King's Buildings, Peter Guthrie Tait Road, Edinburgh EH9 3FD, United Kingdom

${ }^{2}$ Department of Applied Mathematics and Computer Science, Technical University of Denmark, Asmussens Allé, Building 303B, 2800 Kgs. Lyngby, Denmark
\end{abstract}

\author{
a) Author to whom correspondence should be addressed: p.kaklamanos@sms.ed.ac.uk \\ b) nikola.popovic@ed.ac.uk \\ c)krkri@dtu.dk
}

\begin{abstract}
We study a class of multi-parameter three-dimensional systems of ordinary differential equations that exhibit dynamics on three distinct timescales. We apply geometric singular perturbation theory to explore the dependence of the geometry of these systems on their parameters, with a focus on mixed-mode oscillations (MMOs) and their bifurcations. In particular, we uncover a novel geometric mechanism that encodes the transition from MMOs with single epochs of small-amplitude oscillations (SAOs) to those with double-epoch SAOs; the former feature SAOs or pseudo-plateau bursting either "below" or "above" in their time series, while in the latter, SAOs or pseudo-plateau bursting occur both "below" and "above." We identify a relatively simple prototypical three-timescale system that realizes our mechanism, featuring a onedimensional $S$-shaped 2-critical manifold that is embedded into a two-dimensional $S$-shaped critical manifold in a symmetric fashion. We show that the Koper model from chemical kinetics is merely a particular realization of that prototypical system for a specific choice of parameters; in particular, we explain the robust occurrence of mixed-mode dynamics with double epochs of SAOs therein. Finally, we argue that our geometric mechanism can elucidate the mixed-mode dynamics of more complicated systems with a similar underlying geometry, such as a three-dimensional, three-timescale reduction of the Hodgkin-Huxley equations from mathematical neuroscience.
\end{abstract}

Published under an exclusive license by AIP Publishing. https://doi.org/10.1063/5.0073353

Mixed-mode oscillations (MMOs) are trajectories that consist of alternating small-amplitude oscillations (SAOs) and largeamplitude excursions (LAOs). Such trajectories frequently appear in singularly perturbed systems of ordinary differential equations (ODEs) in which the dynamics varies on multiple timescales. While MMOs in low-dimensional two-timescale systems are fairly well-understood and while there has been progress over the past few years on the three-timescale setting, the theory of the latter is still less well-developed. In this work, we propose a simple prototypical three-timescale system, which encodes a geometric mechanism that explains different qualitative types of mixed-mode dynamics and the transition between them, and we classify these types in dependence of the parameters in our system. We show that phenomena that are delicate in the two-timescale setting may become robust in the three-timescale one, and we demonstrate how our results can explain the dynamics of a series of paradigm systems, such as the Koper model from chemical kinetics and the Hodgkin-Huxley equations from mathematical neuroscience.

\section{INTRODUCTION} written as

The Koper model from chemical kinetics ${ }^{17}$ is typically

$$
\begin{gathered}
\varepsilon \dot{x}=k y+3 x-x^{3}-\lambda, \\
\dot{y}=x-2 y+z,
\end{gathered}
$$




$$
\dot{z}=\delta(y-z),
$$

with $k, \lambda \in \mathbb{R}$ and $\varepsilon$ and $\delta$ being real and positive parameters. When $\varepsilon$ is sufficiently small, Eq. (1) exhibits dynamics on two distinct timescales: the variable $x$ is then called the fast variable, while the variables $y$ and $z$ are the slow variables; correspondingly, Eq. (1a) is denoted as the fast equation, whereas (1b) and (1c) are called slow equations. On the other hand, when both $\varepsilon$ and $\delta$ are small, Eq. (1) is a three-timescale system; the variables $x, y$, and $z$ are then called the fast, intermediate, and slow variables, respectively. Correspondingly, Eqs. (1a)-(1c) are denoted as the fast, intermediate, and slow equations, respectively.

Multiple-scale systems of ordinary differential equations frequently feature mixed-mode oscillations (MMOs); ;,5,9,15,17,21,31 these are trajectories that are characterized by an alternation of smallamplitude oscillations (SAOs) and large-amplitude excursions
(LAOs) in the corresponding time series. A particularly fruitful approach for the study of mixed-mode dynamics in singularly perturbed slow-fast systems of the type of the Koper model [Eq. (1)], is based on dynamical systems theory, combining Fenichel's geometric singular perturbation theory $(\mathrm{GSPT})^{10}$ with the desingularization technique known as "blow-up." ${ }^{19}$ Of particular relevance to that approach are localized, non-hyperbolic singularities ("canard points") on the corresponding critical manifolds that generate SAOs in the resulting MMO trajectories, whereas LAOs arise via a global return mechanism along normally hyperbolic portions of those manifolds. A relatively recent, exhaustive review of this so-called "generalized canard mechanism" for the emergence of MMOs can be found in Ref. 6.

Representative MMO trajectories that are realized in the threetimescale Eq. (1) can be seen in Fig. 1, where we set $\varepsilon=0.01=\delta$ throughout. Each such trajectory can be associated with a sequence

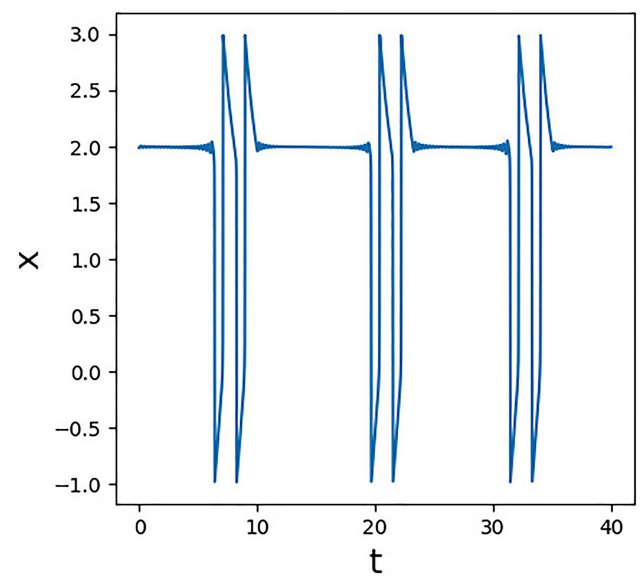

(a)

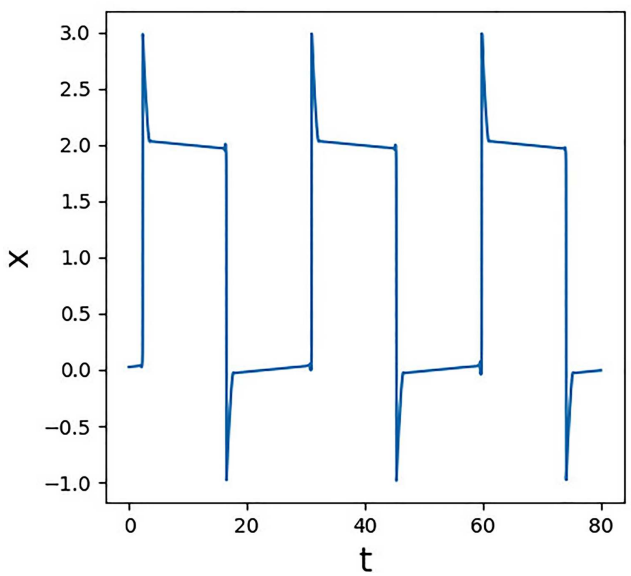

(c)

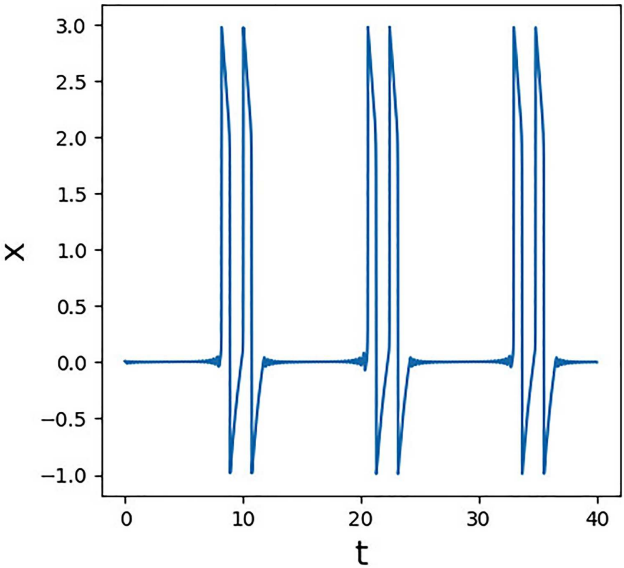

(b)

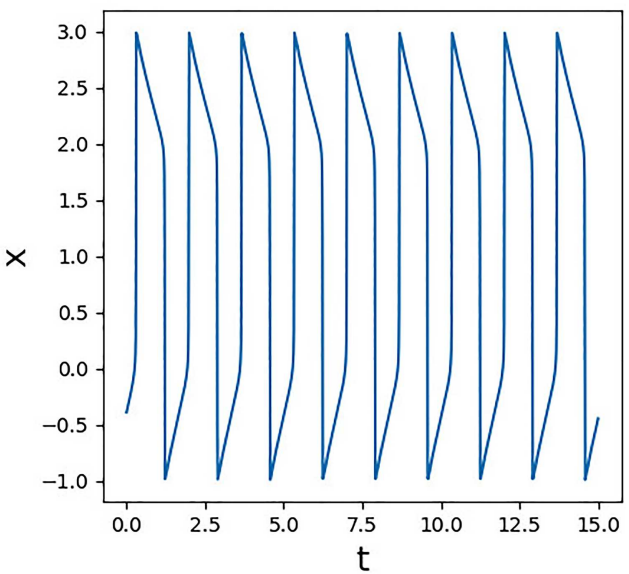

(d)

FIG. 1. Oscillatory dynamics in the Koper model [Eq. (1)] for different values of the parameters $k$ and $\lambda$. (a) MMO trajectory with single epochs of SAOs and Farey sequence $2^{s_{1}} 2^{s_{2}} 2^{s_{3}} \ldots ;$; (b) MMO trajectory with single epochs of SAOs and Farey sequence $2_{s_{1}} 2_{s_{2}} 2_{s_{3}} \cdots ;$; (c) MMO trajectory with double epochs of SAOs and Farey sequence $1^{s_{1}} 1_{s_{2}} s^{s_{3}} 1_{s_{4}} \ldots$; and (d) relaxation oscillation. (a) SAOs "above" ( $\left.k=-4.5, \lambda=-2.0\right)$. (b) SAOs "below" $(k=-4.5, \lambda=2.0)$. (c) Double epochs of SAOs ( $k=-4.0$, $\lambda=0.0$ ). (d) Relaxation oscillation $(k=-4.5, \lambda=0.0)$. 
of the form $\left\{F_{0} F_{1} \cdots\right\}$, called the Farey sequence, which describes the succession of large excursions and small oscillations, where the segments $F_{j}$ are of the form

$$
F_{j}=\left\{\begin{array}{l}
L^{s} \text { if the segment consists of } L \text { LAOs, followed by } s \text { SAOs "above," } \\
L_{s} \text { if the segment consists of } L \text { LAOs, followed by } s \text { SAOs "below." }
\end{array}\right.
$$

If a Farey sequence consists of $L^{s}$-type or $L_{s}$-type segments only, we say that the corresponding MMO trajectory contains single epochs of SAOs or pseudo-plateau bursting, as seen in panels (a) and (b) of Fig. 1, respectively; Farey sequences that consist of both $L^{s}$-type and $L_{s}$-type segments correspond to MMO trajectories that contain double epochs of SAOs or pseudo-plateau bursting, as shown in Fig. 1(c). Finally, relaxation oscillation ${ }^{20}$ refers to oscillatory trajectories that contain large excursions and no SAO segments, i.e., trajectories with associated Farey sequence $\left\{L^{0}\right\}$ [cf. Fig. 1(d)].

MMOs in the Koper model have been extensively studied in the two-timescale context, i.e., for sufficiently small $\varepsilon>0$ and $\delta=\mathcal{O}(1)$ in Eq. (1). ${ }^{6,17,22}$ However, to our knowledge, there are no equivalent studies in the literature for the three-timescale Koper model, with $\varepsilon$ and $\delta$ small in (1), which is the scenario we will consider in this article. In the process, we will uncover a geometric mechanism that encodes bifurcations of MMOs and, in particular, the transition from MMOs with single epochs to double epochs of SAOs therein [recall Fig. 1(c)].

Rather than formulating our mechanism within the framework of the Koper model [Eq. (1)], we will first consider the analytically simpler family of slow-fast systems,

$$
\begin{gathered}
\varepsilon \dot{x}=-y+f_{2} x^{2}+f_{3} x^{3}=: f(x, y), \\
\dot{y}=\alpha x+\beta y-z=: g(x, y, z), \\
\dot{z}=\delta(\mu+\phi(x, y, z))=: \delta h(x, y, z),
\end{gathered}
$$

which can be obtained from (1) via a sequence of affine transformations, with

$$
\begin{gathered}
\varepsilon=\frac{\epsilon}{|k|}, \quad f_{2}=\frac{3}{|k|}, \quad f_{3}=-\frac{1}{|k|}, \\
\alpha=1, \quad \beta=-2, \\
\mu=\frac{k+\lambda+2}{k}, \quad \text { and } \phi(x, y, z)=-y-z .
\end{gathered}
$$

Our motivation for introducing Eq. (2) is twofold: first, the geometry of the Koper model in (1) will turn out to be quite restrictive, as variation of the parameter $k$ in (1a) affects both the associated invariant manifolds and the reduced flow thereon. In (2), on the other hand, the effect of the corresponding parameters $f_{2}, f_{3}$, and $\mu$ on the geometry can be studied independently. Second, it will become apparent that the geometric mechanism described here is generic in that it transcends the Koper model proper; correspondingly, we propose Eq. (2), with $f_{2}>0, f_{3}<0, \alpha, \beta$, and $\mu$ real parameters and $\varepsilon$ and $\delta$ are sufficiently small, as a "prototypical," normal form-type model which encapsulates our mechanism.
Mixed-mode dynamics in three-timescale slow-fast systems of the type in (2) has been studied before (see, e.g., Refs. 3, 4, 6, and 24 for specific examples and further references). In particular, "prototypical" models akin to the one in Eq. (2) have been considered by Krupa et al. ${ }^{18}$ and by Letson et al.; ${ }^{23}$ the corresponding systems of equations are given by

$$
\begin{gathered}
\varepsilon \dot{x}=-y+f_{2} x^{2}+f_{3} x^{3}, \\
\dot{y}=x-z, \\
\dot{z}=\varepsilon(\mu+\phi(x, y, z))
\end{gathered}
$$

and

$$
\begin{gathered}
\varepsilon \dot{x}=y+x^{2}, \\
\dot{y}=-\alpha^{2} x+\beta y+z, \\
\dot{z}=\delta,
\end{gathered}
$$

respectively.

However, it is worth emphasizing that our prototypical model [Eq. (2)], is substantively different from both Eqs. (4) and (5), in spite of the evident similarities between the three systems. Specifically, Eq. (4) refers to the special case of $\alpha=1, \beta=0$, and $\delta=\varepsilon$ in (2). As will become clear in the following, the absence of a linear $y$-term in (4b) makes a crucial difference geometrically, as it is precisely the term that generates MMOs with double SAO epochs in the three-timescale regime. The canonical form in (5), on the other hand, does capture local phenomena and properties of SAOs for $\beta \neq 0$ therein; however, as no cubic $x$-term is present in (5a), it does not allow for LAO-type dynamics via a global return mechanism, nor does it admit true equilibria. In that sense, Eq. (2) combines aspects of both (4) and (5), yielding rich oscillatory behavior which has, to the best of our knowledge, not previously been classified in a three-timescale context.

Correspondingly, our principal aim in this article is a classification of the mixed-mode dynamics in our "generalized prototypical" model [Eq. (2)]. Then, we will apply that classification to the realization thereof that is provided by the Koper model [Eq. (1)], in the three-timescale scenario where $\varepsilon$ and $\delta$ are sufficiently small, on the basis of Fenichel's geometric singular perturbation theory (GSPT) $;{ }^{10}$ the resulting bifurcation diagram, in terms of the parameters $k$ and $\lambda$, is shown in Fig. 2. In particular, we will explain the robust occurrence of mixed-mode dynamics with double epochs of SAOs in the three-timescale Koper model; by contrast, doubleepoch MMOs have only been observed in very narrow parameter regimes in the two-timescale case. ${ }^{6}$ Throughout, we will focus on the novel singular geometry of Eqs. (1) and (2), i.e., on the double 


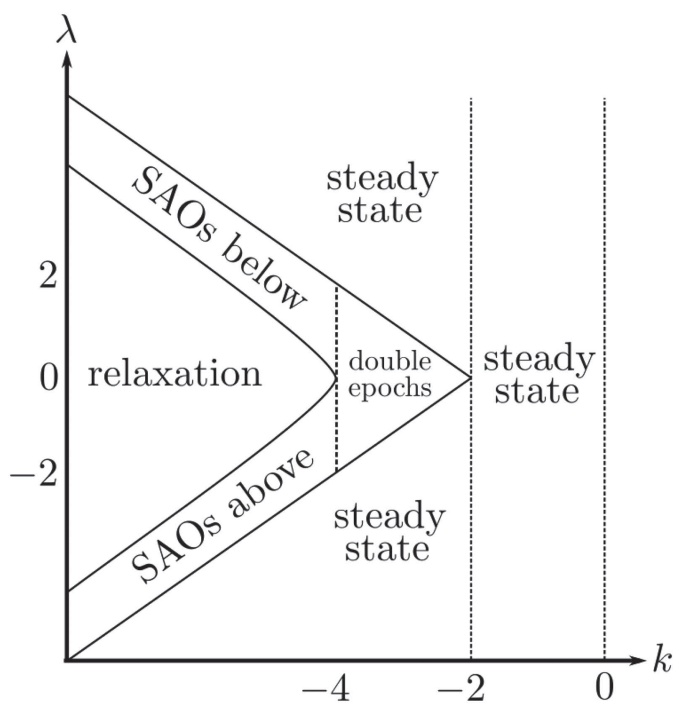

FIG. 2. Two-parameter bifurcation diagram of the three-timescale Koper model [Eq. (1)] to leading order in $\varepsilon$ and $\delta$ (see Sec. IV for details). singular limit of $\varepsilon=0=\delta$ therein, as well as on perturbations off that limit in either $\varepsilon$ or $\delta$. Subsequently, we will comment on the qualitative mixed-mode dynamics, which is expected to result from a full two-parameter perturbation analysis, as is also evidenced by numerical simulation.

Finally, we will argue that the geometric mechanism described here is "generic," in the sense that it allows for the classification of complex mixed-mode dynamics in more complicated systems with similar geometric properties, such as in a three-dimensional reduction of the Hodgkin-Huxley equations from mathematical neuroscience. . $^{914,28}$

This article is organized as follows. In Sec. II, we describe the geometry of the three-time-scale Eq. (2) in the double singular limit of $\varepsilon=0=\delta$ : we define critical and 2-critical manifolds; then, we construct families of singular cycles that form the basis for MMO trajectories of Eq. (2). In Sec. III, we study the singularly perturbed system in (2) for sufficiently small $\varepsilon$ and $\delta$; we classify the mixed-mode dynamics of (2), as illustrated in Fig. 1, by establishing a correspondence with the cycles constructed in Sec. II. In Sec. III, we apply our results to the Koper model from chemical kinetics [Eq. (1)], and we elucidate in detail the structure of the two-parameter bifurcation diagram in Fig. 2. We conclude in Sec. IV with a discussion, and an outlook to future research; in particular, we indicate how our analysis can be extended to a threedimensional reduction of the Hodgkin-Huxley equations derived by Rubin and Wechselberger, ${ }^{28}$ which generalizes our extended prototypical example [Eq. (2)]. Finally, in the Appendix, we provide additional details on SAO-generating mechanisms in the three-timescale context considered here.

\section{THE DOUBLE SINGULAR LIMIT: GEOMETRY AND SINGULAR CYCLES}

In this section, we study the double singular limit of $\varepsilon=0=\delta$ in Eq. (2). To that end, we first describe the singular geometry for $\varepsilon=0$; then, we consider the resulting flow in the limit of $\delta \rightarrow 0$. Finally, we construct singular cycles, which will form the basis of MMO trajectories for Eq. (2) when $\varepsilon$ and $\delta$ are sufficiently small, as considered in Sec. III below.

\section{A. The critical manifold $\mathcal{M}_{1}$}

For sufficiently small $\varepsilon$ and $\delta=\mathcal{O}$ (1) fixed, Eq. (2) is singularly perturbed with respect to the small parameter $\varepsilon$; in particular, (2) describes the dynamics in terms of the intermediate time $t$. Rewriting the governing equations in the fast time $\tau=\frac{t}{\varepsilon}$, we have

$$
\begin{gathered}
x^{\prime}=-y+f_{2} x^{2}+f_{3} x^{3}, \\
y^{\prime}=\varepsilon(\alpha x+\beta y-z), \\
z^{\prime}=\varepsilon \delta(\mu+\phi(x, y, z)),
\end{gathered}
$$

which is a two-timescale system with one fast variable $x$ and two slow variables $y$ and $z$. The reduced problem of the above is obtained by setting $\varepsilon=0$ in (2),

$$
\begin{gathered}
0=-y+f_{2} x^{2}+f_{3} x^{3}, \\
\dot{y}=\alpha x+\beta y-z, \\
\dot{z}=\delta(\mu+\phi(x, y, z)),
\end{gathered}
$$

while the layer problem is found for $\varepsilon=0$ in (6),

$$
\begin{gathered}
x^{\prime}=-y+f_{2} x^{2}+f_{3} x^{3}, \\
y^{\prime}=0, \\
z^{\prime}=0 .
\end{gathered}
$$

We will refer to the flow that is induced by the one-dimensional vector field in Eq. (8) as the fast flow; the corresponding trajectories will be denoted as the fast fibers. The critical manifold $\mathcal{M}_{1}$ for (2) is the set of equilibria for (8), and is given by

$$
\mathcal{M}_{1}:=\left\{(x, y, z) \in \mathbb{R}^{3} \mid f(x, y)=0\right\}=\left\{(x, y, z) \in \mathbb{R}^{3} \mid y=F(x)\right\},
$$

where we define

$$
F(x)=f_{2} x^{2}+f_{3} x^{3} .
$$

The normally hyperbolic portion $\mathcal{S} \subset \mathcal{M}_{1}$ is defined as

$$
\mathcal{S}=\left\{(x, y, z) \in \mathcal{M}_{1} \mid \frac{\partial f}{\partial x}(x, y) \neq 0\right\} .
$$


Then, the manifold $\mathcal{M}_{1}$ can be written as $\mathcal{M}_{1}=\mathcal{S}^{a} \cup \mathcal{S}^{r} \cup \mathcal{F}_{\mathcal{M}_{1}}$, where

$$
\begin{gathered}
\mathcal{S}^{a}=\left\{(x, y, z) \in \mathcal{S} \mid \frac{\partial f}{\partial x}(x, y)<0\right\} \quad \text { and } \\
\mathcal{S}^{r}=\left\{(x, y, z) \in \mathcal{S} \mid \frac{\partial f}{\partial x}(x, y)>0\right\}
\end{gathered}
$$

denote the attracting and repelling sheets of $\mathcal{S}$, respectively, whereas $\mathcal{F}_{\mathcal{M}_{1}}$ is degenerate due to a loss of normal hyperbolicity,

$$
\begin{aligned}
\mathcal{F}_{\mathcal{M}_{1}} & :=\left\{(x, y, z) \in \mathcal{M}_{1} \mid \frac{\partial f}{\partial x}(x, y)=0\right\} \\
& =\left\{(x, y, z) \in \mathcal{M}_{1} \mid x\left(2 f_{2}+3 f_{3} x\right)=0\right\} .
\end{aligned}
$$

In particular, we may write $\mathcal{F}_{\mathcal{M}_{1}}=\mathcal{L}^{-} \cup \mathcal{L}^{+}$, where

$$
\begin{gathered}
\mathcal{L}^{-}=\left\{(x, y, z) \in \mathbb{R}^{3} \mid x=0=y\right\} \quad \text { and } \\
\mathcal{L}^{+}=\left\{(x, y, z) \in \mathbb{R}^{3} \mid x=-\frac{2}{3} \frac{f_{2}}{f_{3}} \text { and } y=\frac{4}{27} \frac{f_{2}^{3}}{f_{3}^{2}}\right\} .
\end{gathered}
$$

Hence, it follows that $\mathcal{S}^{a}=\mathcal{S}^{a^{-}} \cup \mathcal{S}^{a^{+}}$, with

$$
\begin{aligned}
& \mathcal{S}^{a^{-}}=\{(x, y, z) \in \mathcal{S} \mid x<0\} \quad \text { and } \\
& \mathcal{S}^{a^{+}}=\left\{(x, y, z) \in \mathcal{S} \mid x>-\frac{2}{3} \frac{f_{2}}{f_{3}}\right\},
\end{aligned}
$$

while

$$
\mathcal{S}^{r}=\left\{(x, y, z) \in \mathcal{S} \mid 0<x<-\frac{2}{3} \frac{f_{2}}{f_{3}}\right\} .
$$

The normally hyperbolic portion $\mathcal{S}$ of $\mathcal{M}_{1}$, therefore, consists of two attracting sheets $\mathcal{S}^{a^{\mp}}$ and a repelling middle sheet $\mathcal{S}^{r}$ that meets $\mathcal{S}^{a^{\mp}}$ along $\mathcal{L}^{\mp}$, respectively [see Fig. 3(a)]. From the above, it is apparent that $\mathcal{L}^{-}$always coincides with the $z$ axis, whereas variation in $f_{2}$ and $f_{3}$ translates $\mathcal{L}^{+}$, therefore "stretching" or "compressing" $\mathcal{M}_{1}$. (Clearly, variation in $\alpha, \beta$, and $\mu$ has no effect on the geometry

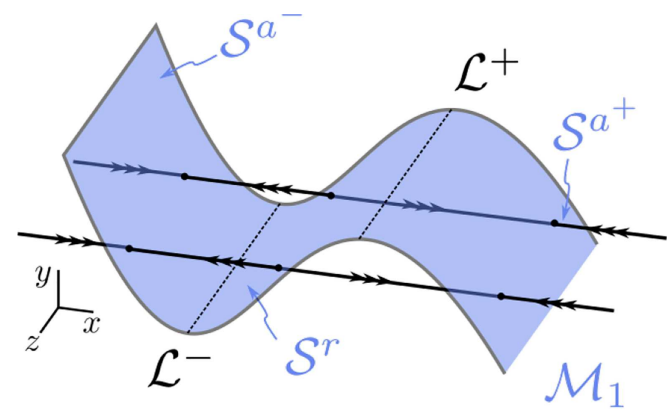

(a) of $\mathcal{M}_{1}$.) Finally, the elements of the sets $\mathcal{Q}^{\mp}$ defined by

$$
\mathcal{Q}^{\mp}=\left\{(x, y, z) \in \mathcal{L}^{\mp} \mid f(x, y)=0=g(x, y, z)\right\}
$$

are called the folded singularities of $\mathcal{M}_{1}$ on $\mathcal{L}^{\mp}$, respectively; ${ }^{29}$ for (2), these sets are the singletons $\mathcal{Q}^{-}=\left\{q^{-}\right\}$and $\mathcal{Q}^{+}=\left\{q^{+}\right\}$, with $q^{\mp}=\left(x_{q}^{\mp}, y_{q}^{\mp}, z_{q}^{\mp}\right)$ located at

$$
x_{q}^{-}=0, \quad y_{q}^{-}=0, \quad \text { and } \quad z_{q}^{-}=0,
$$

as well as at

$$
x_{q}^{+}=-\frac{2 f_{2}}{3 f_{3}}, \quad y_{q}^{+}=\frac{4 f_{2}^{3}}{27 f_{3}^{2}}, \quad \text { and } \quad z_{q}^{+}=\frac{4 \beta f_{2}^{3}}{27 f_{3}^{\beta}}-\frac{2 \alpha f_{2}}{3 f_{3}},
$$

respectively.

Finally, we consider the reduced problem on $\mathcal{M}_{1}$, as given by (7), with $\delta$ being sufficiently small; Eq. (7) is then singularly perturbed with respect to the small parameter $\delta$, written in the intermediate time $t$. To classify the folded singularities $q^{\mp}$ of $\mathcal{M}_{1}$, we project the flow of (7) onto $\mathcal{M}_{1}:{ }^{29}$ recalling that $\mathcal{M}_{1}$ is defined by $f(x, y)=0$, we can apply the chain rule to find

$$
-f_{x} \dot{x}=f_{y} \dot{y},
$$

where $f_{x}=2 f_{2} x+3 f_{3} x^{2}=F^{\prime}(x)$ and $f_{y}=-1$, from (7). We, therefore, obtain

$$
\begin{gathered}
-F^{\prime}(x) \dot{x}=-\alpha x-\beta F(x)+z, \\
\dot{z}=\delta(\mu+\phi(x, F(x), z)),
\end{gathered}
$$

or

$$
\begin{gathered}
\dot{x}=-\alpha x-\beta F(x)+z, \\
\dot{z}=-\delta F^{\prime}(x)(\mu+\phi(x, F(x), z)),
\end{gathered}
$$

after a rescaling of time which introduces a factor of $-F^{\prime}(x)$ on the right-hand sides in (16), reversing the direction of the flow on $\mathcal{S}^{r}$. Folded singularities of Eq. (2) then correspond to equilibria for (17); specifically, for $\delta$ positive, the folded singularities $q^{\mp}$ are folded nodes. ${ }^{23,29}$ Their strong stable manifolds and the fold lines $\mathcal{L}^{\mp}$ define

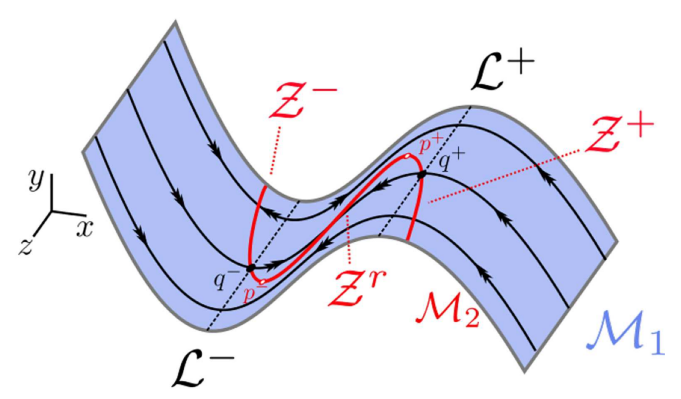

(b)

FIG. 3. Geometry of Eq. (2) in the double singular limit of $\varepsilon=0=\delta$. (a) The critical manifold $\mathcal{M}_{1}$ as the set of equilibria for the fast flow of (8); the fast fibers are parallel to the $x$-direction. (b) The 2-critical manifold $\mathcal{M}_{2}$ as the set of equilibria for the intermediate flow of (21); the intermediate fibers are confined to $\mathcal{M}_{2}$ and evolve on planes with $z$ constant. 
"funnel regions" on the corresponding sheets $\mathcal{S}^{a^{\mp}}$, which essentially determine the basins of attraction to $q^{\mp}$ on $\mathcal{S}^{a^{\mp}} .6,29,32$ Here and in the following, we focus on the flow of Eq. (2) in the vicinity of the fold line $\mathcal{L}^{-}$; with regard to the strong stable manifold of the folded node $q^{-}$, we hence have the following result:

Lemma 1. Let

$$
\mathcal{G}\left(x_{0}, x_{1} ; z_{0} ; \mu\right)=\int_{x_{0}}^{x_{1}} \frac{F^{\prime}(\sigma)\left(\mu+\phi\left(\sigma, F(\sigma), z_{0}\right)\right)}{\alpha \sigma+\beta F(\sigma)-z_{0}} \mathrm{~d} \sigma,
$$

where $F$ is defined as in (10). Then, for sufficiently small $\delta$, the strong stable manifold of the origin for Eq. (17) can be written as the graph

$$
z=\delta \mathcal{G}(0, x ; 0 ; \mu)+\mathcal{O}\left(\delta^{2}\right) \quad \text { for } x \in I^{-},
$$

where $I^{-}$is an appropriately defined, fixed interval about $x=0$.

Proof. Given a trajectory of (17) with initial condition $\left(x_{0}, y_{0}, z_{0}\right)$ on $\mathcal{S}^{a^{\mp}}$, i.e., with $y_{0}=F\left(x_{0}\right)$, let $\xi$ denote the displacement in the $x$-direction of that trajectory under the corresponding flow. Then, in a first approximation, the displacement in the $z$ direction is given by $\delta \mathcal{G}\left(x_{0}, x_{0}+\xi ; z_{0} ; \mu\right)$, where $\mathcal{G}$ is defined as in (18) (see Ref. 18 for details). The result is obtained by setting $x_{0}=0=z_{0}$ in the resulting expression, which corresponds to the unique trajectory of (17) that passes through the origin.

An analogous representation can be obtained for the strong stable manifold of the folded node $q^{+}$. With decreasing $\delta$, the absolute values of the slopes of these strong stable manifolds in the $(x, z)$-plane also decrease; in other words, the manifolds become increasingly horizontal, resulting in the funnels of the folded singularities $q^{\mp}$ becoming "stretched." In the limit of $\delta=0, q^{\mp}$ are (degenerate) folded saddle-nodes (see again Refs. 29 and 23 for details). For future reference, we note that the associated strong manifolds ("strong canards") correspond to the unique intermediate fibers on $\mathcal{S}^{a^{\mp}}$ that cross $q^{\mp}$, respectively, while the corresponding weak manifolds ("weak canards") can be locally approximated by the 2-critical manifold $\mathcal{M}_{2}$, which is introduced in Sec. II B.

\section{B. The 2-critical manifold $\mathcal{M}_{2}$}

We can view the differential-algebraic system in (7), as well as its desingularized counterpart in (17), as slow-fast vector fields on $\mathcal{M}_{1}$. The layer problem corresponding to (7), therefore, reads

$$
\begin{gathered}
0=-y+F(x), \\
\dot{y}=\alpha x+\beta y-z, \\
\dot{z}=0,
\end{gathered}
$$

or

$$
\begin{gathered}
0=-y+F(x), \\
-F^{\prime}(x) \dot{x}=-\alpha x-\beta F(x)+z, \\
\dot{z}=0 .
\end{gathered}
$$

We will refer to the above as the intermediate flow, and to the corresponding trajectories as the intermediate fibers [see panel (b) of
Fig. 3]. We emphasize that the intermediate flow is not defined on the fold lines $\mathcal{L}^{\mp}$, whereon $F^{\prime}(x)=0$.

Rewriting Eq. (2) in the slow time $s=\delta t$, we have

$$
\begin{gathered}
\varepsilon \delta x^{\prime}=-y+F(x), \\
\delta y^{\prime}=\alpha x+\beta y-z, \\
z^{\prime}=\mu+\phi(x, y, z) .
\end{gathered}
$$

The reduced system that is obtained from (22) is given by

$$
\begin{gathered}
0=-y+F(x), \\
0=\alpha x+\beta F(x)-z, \\
z^{\prime}=\mu+\phi(x, F(x), z),
\end{gathered}
$$

which we will refer to as the slow flow of Eq. (2). The 2-critical manifold (to the best of our knowledge, it seems that there is no generally agreed terminology for $\mathcal{M}_{2}$ yet; in Refs. 3 and 23, it is called "superslow," although it is defined in the double singular limit, whereas it is denoted as the "2-critical" manifold in Ref. 1, which is also the term adopted here.) $\mathcal{M}_{2}$ is defined as

$$
\begin{aligned}
\mathcal{M}_{2} & :=\left\{(x, y, z) \in \mathbb{R}^{3} \mid f(x, y)=0=g(x, y, z)\right\} \\
& =\left\{(x, y, z) \in \mathcal{M}_{1} \mid z=G(x)\right\},
\end{aligned}
$$

where we write

$$
G(x)=\alpha x+\beta F(x) .
$$

Away from $\mathcal{L}^{\mp}, \mathcal{M}_{2}$ is the set of equilibria for (21). The reduced flow on $\mathcal{M}_{2}$ is then given by

$$
G^{\prime}(x) x^{\prime}=\mu+\phi(x, F(x), G(x)) .
$$

In the above notation, the coordinates of the folded singularities in (15) can be expressed as $y_{q}^{\mp}=F\left(x_{q}^{\mp}\right)$ and $z_{q}^{\mp}=G\left(x_{q}^{\mp}\right)$; in particular, we have that

$$
Q^{\mp}=\mathcal{M}_{2} \cap \mathcal{L}^{\mp} .
$$

The manifold $\mathcal{M}_{2}$ can be written as the union $\mathcal{M}_{2}=\mathcal{Z} \cup$ $\mathcal{F}_{\mathcal{M}_{2}}$, where

$$
\mathcal{Z}=\left\{(x, y, z) \in \mathcal{M}_{2} \mid \frac{d g}{d x}(x, F(x), G(x)) \neq 0\right\}
$$

is normally hyperbolic and the set

$$
\begin{aligned}
\mathcal{F}_{\mathcal{M}_{2}} & :=\left\{(x, y, z) \in \mathcal{M}_{2} \mid \frac{d g}{d x}(x, F(x), G(x))=0\right\} \\
& =\left\{(x, y, z) \in \mathcal{M}_{2} \mid \alpha+2 \beta f_{2} x+3 \beta f_{3} x^{2}=0\right\}
\end{aligned}
$$

is degenerate. Equation (28) yields $\mathcal{F}_{\mathcal{M}_{2}}=\left\{p^{-}, p^{+}\right\}$, with

$$
p^{\mp}=\left\{(x, y, z) \in \mathcal{M}_{2} \mid x=x_{p}^{\mp}\right\},
$$

where

$x_{p}^{\mp}=\frac{-\beta f_{2} \pm \sqrt{\beta^{2} f_{2}^{2}-3 \alpha \beta f_{3}}}{3 \beta f_{3}}, \quad y_{p}^{\mp}=F\left(x_{p}^{\mp}\right), \quad$ and $\quad z_{p}^{\mp}=G\left(x_{p}^{\mp}\right)$. 
The non-hyperbolic points $p^{\mp}$ are called the fold points of $\mathcal{M}_{2}$. Equation (30) immediately implies:

Proposition 1. The manifold $\mathcal{M}_{2}$ admits

1. exactly two fold points if and only if $\beta^{2} f_{2}^{2}-3 \alpha \beta f_{3}>0$;

2. exactly one non-hyperbolic point if and only if $\beta^{2} f_{2}^{2}-3 \alpha \beta f_{3}=0$; and

3. no non-hyperbolic points if and only if $\beta^{2} f_{2}-3 \alpha \beta f_{3}<0$.

Remark 1. Under the conditions stated in Proposition 1, the fold points $p^{\mp}$ of $\mathcal{M}_{2}$ are "inherited" from the fold lines $\mathcal{L}^{\mp}$ of $\mathcal{M}_{1}$, in the sense that $G(x)$ in (24) is a cubic polynomial because $F(x)$ in (9) is.

We note that a necessary (but not sufficient) condition for $\mathcal{M}_{2}$ to have two fold points is the requirement that $\beta \neq 0$. If $\mathcal{M}_{2}$ admits two fold points, then the normally hyperbolic portion $\mathcal{Z}$ of $\mathcal{M}_{2}$ consists of three branches: $\mathcal{Z}=\mathcal{Z}^{-} \cup \mathcal{Z}^{0} \cup \mathcal{Z}^{+}$, where

$$
\begin{gathered}
\mathcal{Z}^{-}=\left\{(x, y, z) \in \mathcal{Z} \mid x<x_{p}^{-}\right\}, \\
\mathcal{Z}^{0}=\left\{(x, y, z) \in \mathcal{Z} \mid x_{p}^{-}<x<x_{p}^{+}\right\}, \quad \text { and } \\
\mathcal{Z}^{+}=\left\{(x, y, z) \in \mathcal{Z} \mid x>x_{p}^{+}\right\} .
\end{gathered}
$$

A rescaling of time in $(21)$ by a factor of $-F^{\prime}(x)$, as was done in (17), reverses the orientation on $\mathcal{S}^{r}$, whereas it preserves it on $\mathcal{S}^{a^{\mp}}$. If the 2-critical manifold $\mathcal{M}_{2}$ admits two fold points, i.e., if $\beta^{2} f_{2}^{2}-3 \alpha \beta f_{3}>0$ by Proposition 1, then the portion of the middle branch $\mathcal{Z}^{0} \cap \mathcal{S}^{r}$ of $\mathcal{M}_{2}$ in (31) is attracting, respectively, repelling, under the flow of the desingularized Eq. (21) for $\beta<0$, respectively, $\beta>0$. That is, the stability properties of $\mathcal{Z}$ within $\mathcal{S}$-i.e., within $\mathcal{M}_{1}$ and away from $\mathcal{L}^{\mp}$-are determined from the scalar equation $\dot{x}=-\alpha x-\beta F(x)+z$, with the stability of $\mathcal{Z}^{0}$ being reversed on $\mathcal{S}^{r}$ (cf. Fig. 4). It follows that $\mathcal{Z}^{0}$ could potentially be separated into attracting portions in $\mathcal{S}^{r}$ and repelling ones in $\mathcal{S}^{a^{\mp}}$ [cf. Fig. 4(a)],

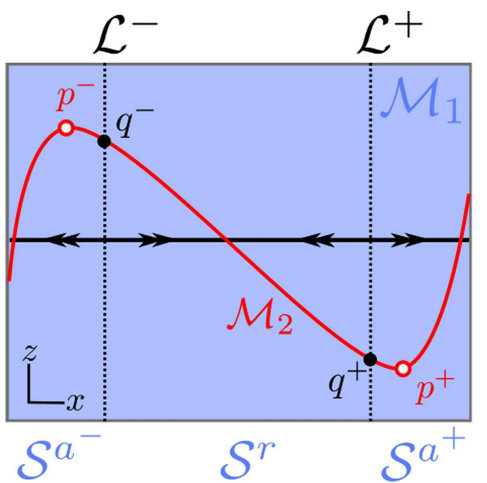

(a)

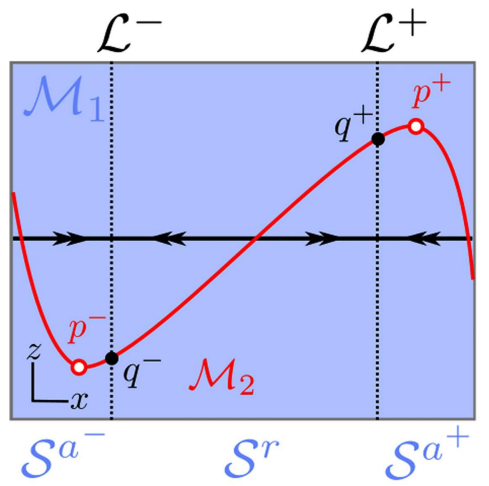

(d)

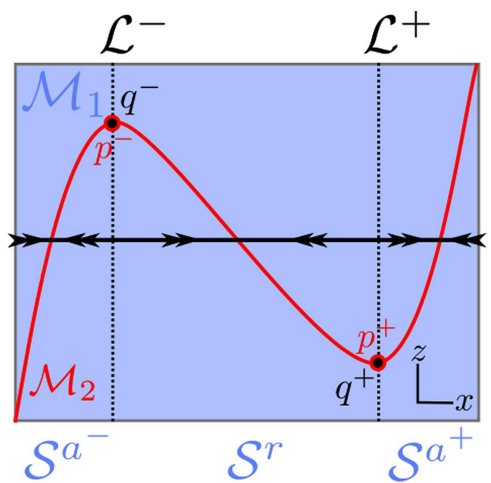

(b)

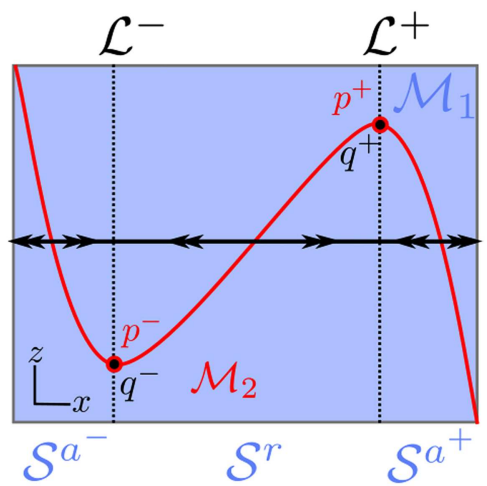

(e)

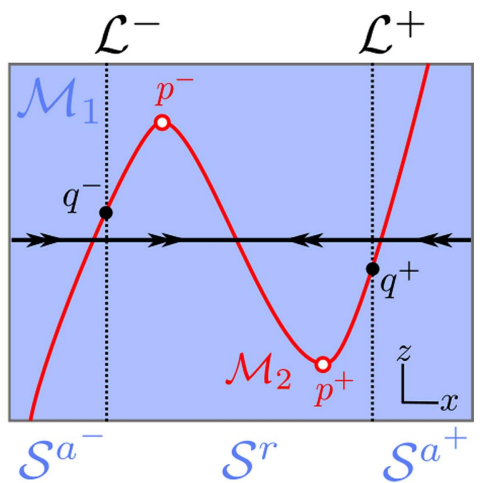

(c)

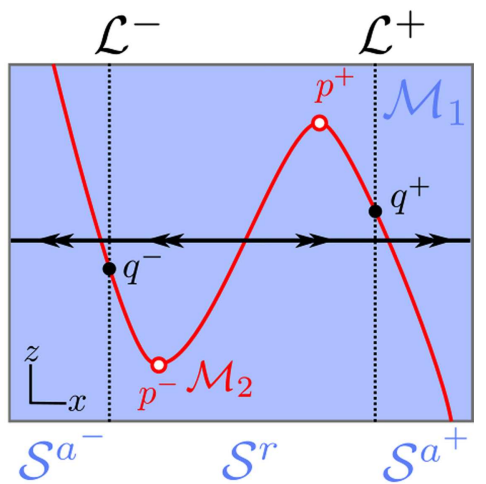

(f)

FIG. 4. Projection of the 2-critical manifold $\mathcal{M}_{2}$ and of the fold lines $\mathcal{L}^{\mp}$ of the critical manifold $\mathcal{M}_{1}$ onto the $(x, z)$-plane: in dependence on the parameters $\alpha$ and $\beta$, the pair of fold points $p^{\mp}$ of $\mathcal{M}_{2}$ lies either on $\mathcal{S}^{r}$ [panels (c) and (f)], on $\mathcal{S}^{a^{\mp}}$ [panels (a) and (d)], or on $\mathcal{L}^{\mp}$ [panels (b) and (e)]. (a) $\beta<0, \alpha<0$. (b) $\beta<0, \alpha=0$. (c) $\beta<0, \alpha>0$. (d) $\beta>0, \alpha<0$. (e) $\beta>0, \alpha=0$. (f) $\beta>0, \alpha>0$. 
or vice versa [see Fig. 4(d)]. A similar argument applies to the outer branches $\mathcal{Z}^{\mp}$, as seen in panels (c) and (f) of Fig. 4.

Remark 2. We remark that the above discussion of the stability of $\mathcal{Z}$ in the double singular limit of $\varepsilon=0=\delta$ is alternative to the approach outlined in Ref. 1 , where $x$ is expressed as a function of $y$ in (20b) via the algebraic constraint in (20a), as well as to that in Ref. 23, where only the stability of the partially perturbed counterpart of $\mathcal{Z}$ is investigated [see the Appendix for an extension of the latter within the framework of Eq. (2)].

Remark 3. By the above, the folded singularities $q^{\mp}$ of $\mathcal{M}_{1}$ are located at the intersections between $\mathcal{M}_{2}$ and $\mathcal{L}^{\mp}$. In the double singular limit of $\varepsilon=0=\delta$, the points $q^{\mp}$ coincide with the folded singularities of $\mathcal{M}_{1}$ for $\varepsilon=0$ and $\delta=\mathcal{O}(1)$, i.e., in the two-timescale limit, which stems from the fact that the fast and intermediate equations (2a) and (2b) do not depend on $\delta$ in our case.

\section{Relative geometry}

In this subsection, we describe the position of the folded singularities $q^{\mp}$ of $\mathcal{M}_{1}$ relative to each other, as well as of the fold points $p^{\mp}$ of $\mathcal{M}_{2}$-assuming that a pair of such points exists-relative to the fold lines $\mathcal{L}^{\mp}$.

Proposition 2. Assume that $\mathcal{M}_{2}$ admits two fold points, i.e., that $\beta^{2} f_{2}^{2}-3 \alpha \beta f_{3}>0$, by Proposition 1 .

1. If $\alpha \beta<0$, then both fold points of $\mathcal{M}_{2}$ lie on $\mathcal{S}^{r}$;

2. if $\alpha \beta>0$, then one fold point of $\mathcal{M}_{2}$ lies on $\mathcal{S}^{a^{-}}$, while the other fold point lies on $\mathcal{S}^{a^{+}}$; and

3. if $\alpha=0$, then one fold point of $\mathcal{M}_{2}$ lies on $\mathcal{L}^{-}$, while the other fold point lies on $\mathcal{L}^{+}$.

Proof. The result follows from a comparison of the values of $x^{\mp}$ in (30) with the $x$-coordinates of $\mathcal{L}^{\mp}$ in the three cases where $\alpha \beta<0$, $\alpha \beta>0$, and $\alpha=0$, respectively.

Proposition 2 is again summarized in Fig. 4. We remark that the symmetry described therein breaks down when $\mathcal{O}\left(x^{2}\right)$-terms are included in the intermediate Eq. (2b) (see Refs. 7 and 25 for some examples). That symmetry is relevant to the example of the Koper model studied here, as well as to the multi-timescale Hodgkin-Huxley equations which are analyzed in the upcoming work, Ref. 16 (we remark on the asymmetric case in our discussion in Sec. $V$ below). If $\beta=0$, then the projection of the critical manifold $\mathcal{M}_{2}$ onto the $(x, z)$-plane is a straight line. That case has been studied in Refs. 3, 4, and 18 [recall also Eq. (4)].

We now turn our attention to the location of the folded singularities of $\mathcal{M}_{1}$ relative to each other and with respect to the fast and intermediate fibers defined previously (recall Fig. 3). We first define planes that contain the folded singularities and that are perpendicular to the fold lines $\mathcal{L}^{-}$and $\mathcal{L}^{+}$, as follows.

Definition 1. Denote by $\mathcal{P}^{\mp}$ the planes $\mathcal{P}^{\mp}=\{(x, y, z)$ $\left.\in \mathbb{R}^{3} \mid z=z_{q}^{\mp}\right\}$, where $z_{q}^{\mp}$ are the $z$-coordinates of the folded singularities $q^{\mp}$ of $\mathcal{M}_{1}$ on $\mathcal{L}^{\mp}$, respectively. We will refer to $\mathcal{P}^{\mp}$ as normal planes in the following.
Definition 2. The folded singularities $q^{\mp}$ of $\mathcal{M}_{1}$ are said to be

1. aligned if $\mathcal{P}^{-} \equiv \mathcal{P}^{+}$

2. connected if they are not aligned and if $\mathcal{P}^{\mp} \cap \mathcal{Z}^{ \pm} \neq \emptyset$; or

3. remote if they are neither aligned nor connected, i.e., if $\mathcal{P}^{-} \not \equiv \mathcal{P}^{+}$ and $\mathcal{P}^{\mp} \cap \mathcal{Z}^{ \pm}=\emptyset$.

In dependence of the parameters $\alpha, \beta, f_{2}$, and $f_{3}$ in Eq. (2), we have the following result on the position of $q^{-}$and $q^{+}$relative to each other: above.

Proposition 3. Recall the classification in Definition 2

1. For $\alpha \beta<0$, the folded singularities $q^{\mp}$ of $\mathcal{M}_{1}$ are aligned if $\frac{\alpha}{\beta}$ $=\frac{2 f_{2}^{2}}{9 f_{3}}$, connected if $\frac{\alpha}{\beta}>\frac{2 f_{2}^{2}}{9 f_{3}}$, and remote if $\frac{\alpha}{\beta}<\frac{2 f_{2}^{2}}{9 f_{3}}$.

2. For $\alpha \beta \geq 0$ with $\beta \neq 0$, the folded singularities $q^{\mp}$ are connected.

3. For $\beta=0$ with $\alpha \neq 0$, the folded singularities $q^{\mp}$ are remote.

Proof. The statements follow from Eq. (15) and the properties of $G(x)$ in (25); see panels (c) and (d) of Fig. 4 for cases corresponding to the first statement, and panels (a), (b), (e), and (f) for cases corresponding to the second statement.

In what is to come, we will restrict our attention to the case that is illustrated in panel (c) of Fig. 4:

Assumption 1. In the following, we assume that $\alpha>0$ and $\beta<0$ in Eq. (2).

Assumption 1 is made for three reasons. First, it is consistent with the Koper model [Eq. (1)] after transformation to the prototypical Eq. (2) [in particular, it follows that the scenarios illustrated in panels (b) and (e) of Fig. 4 cannot be realized in (1)]. Second, given $\beta \neq 0$, remote singularities can only be present when $\alpha \beta<0$. Third, given Assumption 1, the outer branches $\mathcal{Z}^{\mp} \cap \mathcal{S}^{a \mp}$ of $\mathcal{Z}$ are attracting, while the middle branch is repelling, which allows for the construction of closed singular periodic orbits ("cycles") which will serve as templates for the corresponding MMO trajectories, as will become apparent in Sec. II D. In particular, since $\mathcal{Z}^{0}$ is entirely contained in $\mathcal{S}^{r}$, we will write

$$
\mathcal{Z}=\mathcal{Z}^{-} \cup \mathcal{Z}^{r} \cup \mathcal{Z}^{+}
$$

in the following.

\section{Singular cycles}

We now consider the reduced flow on $\mathcal{M}_{2}$. We impose the following assumption on the function $\phi(x, y, z)$ in the slow Eq. (2c):

Assumption 2. The function $\phi(x, y, z)$ in Eq. (2c) is such that $\phi\left(x_{q}^{-}, y_{q}^{-}, z_{q}^{-}\right)=0, \phi(x, F(x), G(x))>0$ for $x<x_{q}^{-}, \phi\left(x_{q}^{+}, y_{q}^{+}, z_{q}^{+}\right)$ $\leq 0$, and $\phi(x, F(x), G(x))<0$ for $x>x_{q}^{+}$.

Assumption 2 ensures that the reduced flow under (26) on the portions $\mathcal{Z}^{\mp} \cap \mathcal{S}^{a \mp}$ is directed toward the folded singularities $q^{\mp}$ of $\mathcal{M}_{1}$. We emphasize that the properties of that flow, therefore, crucially depend on $\mu$ : in particular, we have that for $\mu=0$, a true global equilibrium of Eq. (2) coincides with $q^{-}$(see Sec. III).

Assumptions 1 and 2 together imply the existence of singular cycles in Eq. (2), the properties of which are determined by the relative position of the folded singularities $q^{\mp}$ of $\mathcal{M}_{1}$, as classified in Proposition 3. [The choice of $\phi(x, y, z)$ in (2c) does not affect 
that classification provided that Assumption 2 is satisfied; correspondingly, we do not specify it here.] These cycles are defined as the concatenation of singular orbits for the corresponding limiting systems in (8), (21), and (23), respectively. hold.

Proposition 4. Assume that Assumption 1 and Assumption 2

1. If the folded singularities $q^{\mp}$ of $\mathcal{M}_{1}$ are remote, then there exist a singular cycle evolving on $\mathcal{P}^{-}$, a singular cycle evolving on $\mathcal{P}^{+}$, and a family of singular cycles in between; each of the cycles in that family evolves on a plane parallel to $\mathcal{P}^{\mp}$ that lies between $\mathcal{P}^{-}$and $\mathcal{P}^{+}$. These cycles are "two-scale," in the sense that the singular dynamics on them alternates between the fast and the intermediate timescale (on $\mathcal{M}_{1} \backslash \mathcal{M}_{2}$ ).

2. If $q^{\mp}$ are aligned, then there exists exactly one singular cycle that evolves on the plane $\mathcal{P}:=\mathcal{P}^{-} \equiv \mathcal{P}^{+}$. This cycle is "two-scale," in the sense that the singular dynamics on it alternates between the fast and the intermediate timescale (on $\mathcal{M}_{1} \backslash \mathcal{M}_{2}$ ).

3. If $q^{\mp}$ are connected, then there exists exactly one singular cycle that evolves on a subset of $\mathcal{P}^{-} \cup \mathcal{Z}^{-} \cup \mathcal{P}^{+} \cup \mathcal{Z}^{+}$. This cycle is "three-scale," in the sense that the singular dynamics on it alternates between the fast, the intermediate (on $\mathcal{M}_{1} \backslash \mathcal{M}_{2}$ ), and the slow timescale (on $\mathcal{M}_{2}$ ).

Definition 2 and Proposition 4 are summarized in Fig. 5, where we recall that the fast, intermediate, and slow dynamics is given by the limiting systems in (8), (21), and (23), respectively.

\section{SINGULAR PERTURBATION}

In this section, we discuss the correspondence between the families of singular cycles constructed in Proposition 4 and the MMO trajectories that perturb from those cycles for $\varepsilon$ and $\delta$ being positive, but sufficiently small, in Eq. (2). In the process, we give a qualitative characterization of the resulting mixed-mode dynamics in dependence of system parameters.

By standard GSPT, ${ }^{1,10,19}$ we obtain slow manifolds $\mathcal{S}_{\varepsilon \delta}^{a, r}$ as perturbations of $\mathcal{S}^{a, r}$ away from the fold lines $\mathcal{L}^{\mp}$, for sufficiently small $\varepsilon, \delta>0$. The dynamics on these locally invariant manifolds is itself slow-fast with respect to the singular perturbation parameter $\delta$, which implies the existence of super-slow locally invariant manifolds $\mathcal{Z}_{\varepsilon \delta}^{\mp, r}$ as perturbations of $\mathcal{Z}^{\mp, r}$ away from the fold points $p^{\mp}$. In particular, the manifolds $\mathcal{Z}_{\varepsilon \delta}^{\mp}$ locally approximate the weak canards of the folded singularities $q^{\mp}$, respectively ${ }^{23}$ [recall (17)].

First, we remark on the transition between steady-state behavior and oscillatory dynamics in Eq. (2) in dependence of $\mu$. For $\varepsilon=0=\delta$, true equilibria of (2) cross the folded singularities $q^{\mp}$ at

$$
\mu_{q}^{-}=-\phi\left(x_{q}^{-}, y_{q}^{-}, z_{q}^{-}\right) \text {and } \mu_{q}^{+}=-\phi\left(x_{q}^{+}, y_{q}^{+}, z_{q}^{+}\right) \text {, }
$$

where we recall that $\left(x_{q}^{-}, y_{q}^{-}, z_{q}^{-}\right)=(0,0,0)$ and, hence, that $0=\mu_{q}^{-}<\mu_{q}^{+}$, by Assumption 2 .

By Refs. 6, 11, and 18, Hopf bifurcations occur $\mathcal{O}(\varepsilon, \delta)$-close to $\mu_{q}^{\mp}$ for sufficiently small $\varepsilon, \delta>0$ in Eq. (2); when these are supercritical, they generate small-amplitude limit cycles in the vicinity of the folded singularities $q^{\mp}$, which correspond to MMOs with signature $0^{k}$. The global mixed-mode dynamics that may arise due to those Hopf bifurcations does not seem to be well-understood in the three-scale regime considered here ${ }^{6}$ However, MMO trajectories that contain both SAO and LAO segments have been shown to emerge either in a "slow passage through a canard explosion" that occurs $\mathcal{O}(\varepsilon, \delta)$-away from the corresponding Hopf points ${ }^{18,23}$ or via a delayed Hopf-type phenomenon on $\mathcal{Z}_{\varepsilon \delta}^{\mp} ;, 4,23$ the underlying mechanisms are briefly addressed in the Appendix in the context of Eq. (2). Crucially, for fixed $\mu \in\left(\mu_{q}^{-}, \mu_{q}^{+}\right)$, one can find $\varepsilon, \delta>0$ sufficiently small such that (2) features global mixed-mode dynamics, which we will study in dependence of the parameters $f_{2}, f_{3}, \alpha$, and $\beta$ therein. For future reference, we will write

$$
M:=\left(\mu_{q}^{-}, \mu_{q}^{+}\right) \quad \text { and } \quad \bar{M}:=\left[\mu_{q}^{-}, \mu_{q}^{+}\right] .
$$

For $\varepsilon$ and $\delta$ positive and sufficiently small in (2), trajectories can hence be composed from components that evolve close to fast, intermediate, and slow segments of the corresponding singular cycles introduced in Sec. II. In a first approximation, where the fast and intermediate segments are approximated as straight lines-the latter in the $(x, z)$-plane-trajectories are attracted to the vicinities of both folded singularities $q^{\mp}$ if these are aligned or connected, whereas they tend to either $q^{-}$or $q^{+}$if the singularities are remote, as can be seen from Fig. 5. [In Sec. II, we showed that the funnels of the folded nodes $q^{\mp}$ for Eq. (2) expand with decreasing $\delta$; in the three-timescale limit as $\delta$ approaches zero, these funnels can be viewed as having been "stretched" in one direction.] From the well-established theory of two-timescale singular perturbations, it is known that SAOs arise in the passage past folded singularities under the perturbed flow (see Refs. 4, 6, and 32); the underlying local two-timescale mechanisms are well-understood. Again, we discuss their three-timescale analogs in Subsection 1 in the Appendix. In particular, we conclude that SAOs are observed "above" or "below," in the language of Fig. 1, depending on which folded singularity of Eq. (2) trajectories are attracted to; double epochs of SAOs can occur when trajectories are attracted to both folded singularities $q^{\mp}$. The mixed-mode dynamics of Eq. (2) can hence naturally be classified according to whether the folded singularities $q^{\mp}$ are aligned, connected, or remote (cf. Fig. 6).

\section{A. Aligned or connected singularities}

When the folded singularities of $\mathcal{M}_{1}$ are aligned or connected, "double epochs" of slow dynamics are observed in (2) for sufficiently small $\varepsilon, \delta>0$ and $\mu \in M$ (see Figs. 6 and 7).

Theorem 1. Assume that Assumptions 1 and 2 hold, that the folded singularities of Eq. (2) are aligned or connected in the sense of Definition 2, i.e., that $\frac{\alpha}{\beta} \geq \frac{2 f_{2}^{2}}{9 f_{3}}$ in (2), and fix $\mu \in M$. Then, there exist sufficiently small $\varepsilon_{0}, \delta_{0}>0$ such that (2) features MMOs with double epochs of perturbed slow dynamics for all $(\varepsilon, \delta) \in\left(0, \varepsilon_{0}\right) \times\left(0, \delta_{0}\right)$.

Proof. We need to show that, under the stated assumptions, Eq. (2) admits oscillatory trajectories that consist of repeat sequences of fast, intermediate, and slow segments near $\mathcal{S}^{a^{-}}$, followed by fast, intermediate, and slow segments near $\mathcal{S}^{a^{+}}$(see Fig. 7). To that end, we define the sections 


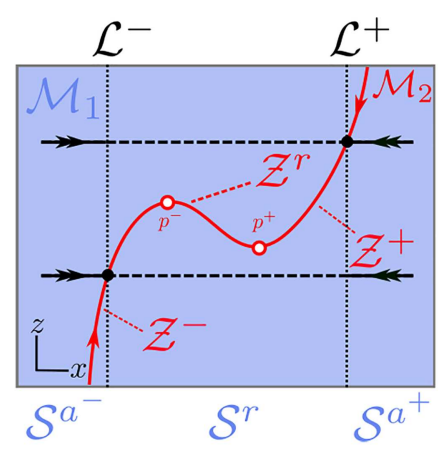

(a)

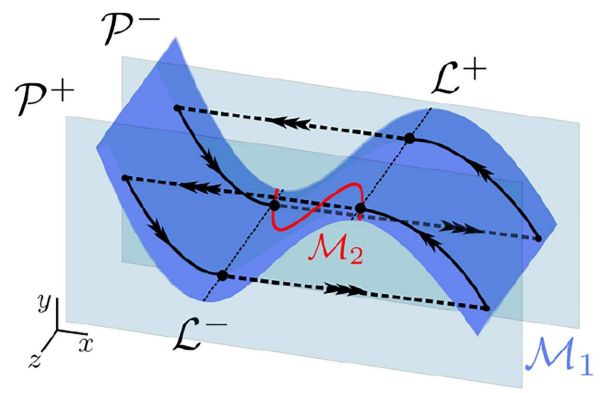

(d)

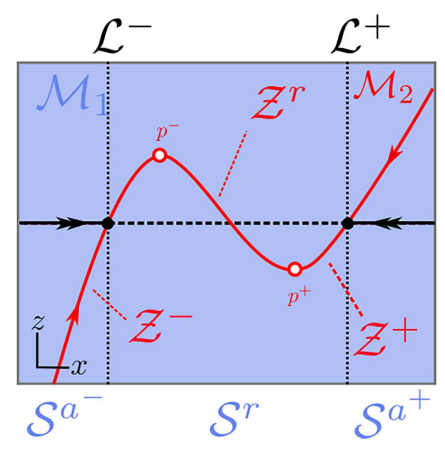

(b)

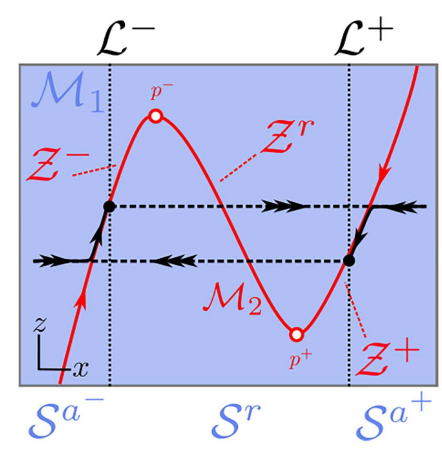

(c)

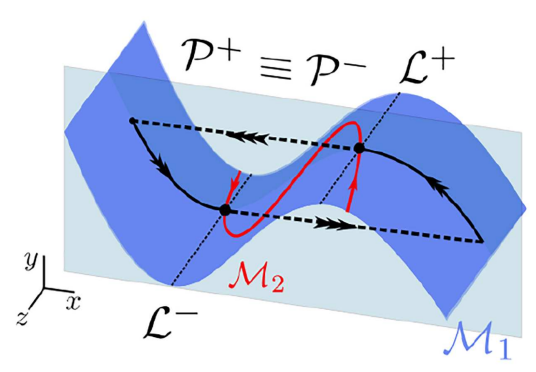

(e)

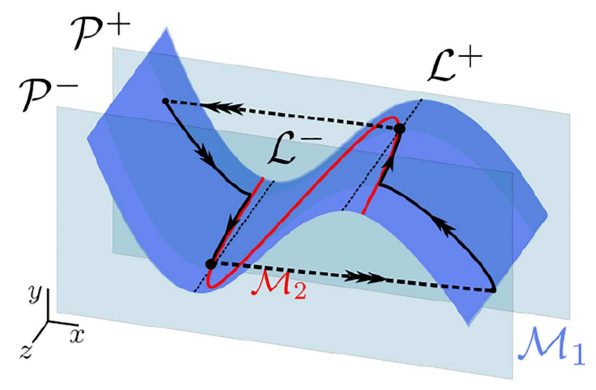

(f)

FIG. 5. Relative geometry of the folded singularities $q^{\mp}$ of $\mathcal{M}_{1}$ according to Definition 2 (top row); bifurcation of the resulting singular cycles, as described in Proposition 4 (bottom row). (a) Remote singularities. (b) Aligned singularities. (c) Connected singularities. (d) Family of "two-scale" cycles. (e) Unique "two-scale" cycle. (f) Unique "three-scale" cycle.

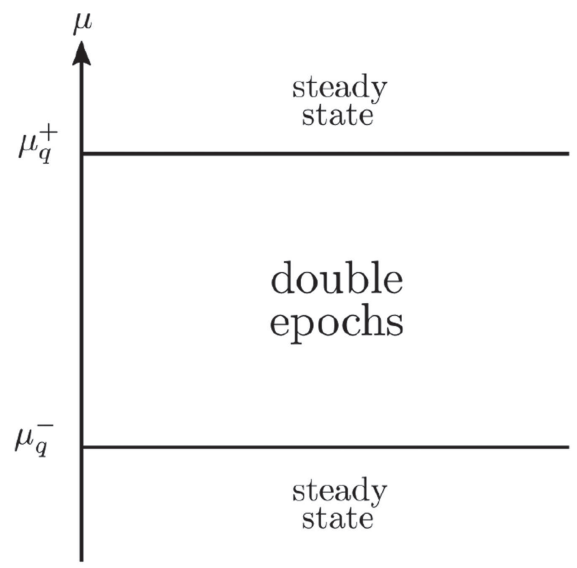

(a)

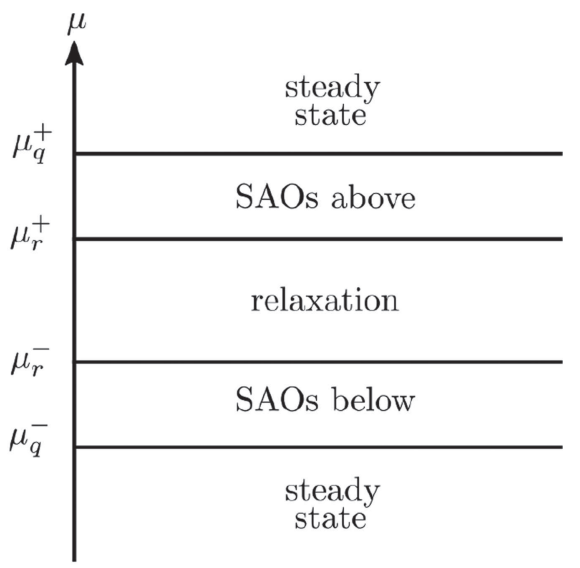

(b)

FIG. 6. Dynamics of Eq. (2), with $\mathrm{f}_{2}, \mathrm{f}_{3}, \alpha$, and $\beta$ fixed and $\varepsilon, \delta>0$ being sufficiently small: the $\mu$-values $\mu_{\alpha}^{\mp}$ distinguish between oscillatory dynamics and steady-state behavior. (a) When $\frac{\alpha}{\beta} \geq \frac{2 f_{2}^{2}}{9 f_{3}}$, the singular geometry of (2) is such that double epochs of perturbed slow dynamics occur for sufficiently small $\varepsilon, \delta>0$. (b) When $\frac{\alpha}{\beta}<\frac{2 f_{2}^{2}}{9 f_{3}}$, there exist two values $\mu_{r}^{\mp}$ that separate MMO trajectories with single epochs of SAOs from relaxation oscillation, in dependence of the properties of $\phi(x, y, z)$ in $(2 \mathrm{c})$. (a) Aligned or connected singularities $\left(\frac{\alpha}{\beta} \geq \frac{2 f_{2}^{2}}{9 f_{3}}\right)$. (b) Remote singularities $\left(\frac{\alpha}{\beta}<\frac{2 f_{2}^{2}}{9 f_{3}}\right)$. 


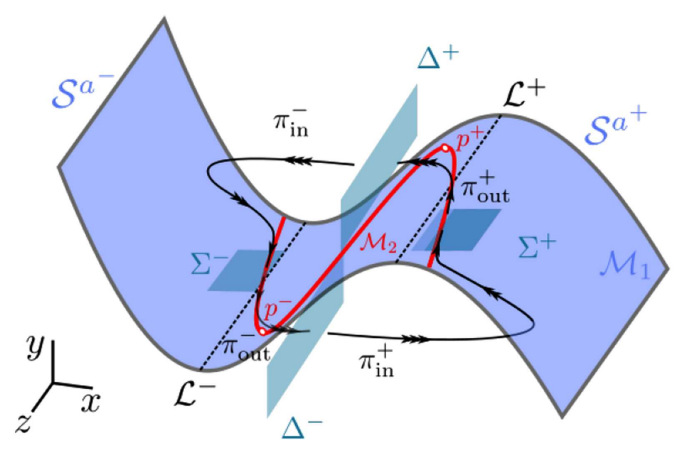

(a)

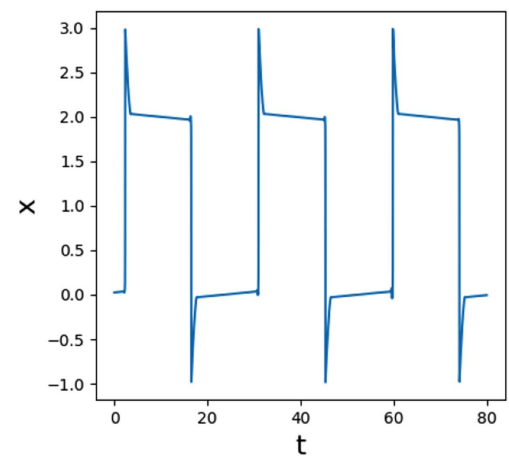

(b)

FIG. 7. Schematic illustration of the emergence of MMO trajectories with double epochs of perturbed slow dynamics in (2) for the case of aligned or connected singularities: (a) singular geometry and transition maps and (b) corresponding time series for the Koper model, as defined in Sec. IV.

$$
\begin{aligned}
& \Delta^{+}=\left\{(x, y, z) \in \mathbb{R}^{3} \mid x=\frac{x_{q}^{+}+x_{q}^{-}}{2}, y>\frac{y_{q}^{+}+y_{q}^{-}}{2}, \text { and } z>\frac{z_{q}^{+}+z_{q}^{-}}{2}\right\}, \\
& \Delta^{-}=\left\{(x, y, z) \in \mathbb{R}^{3} \mid x=\frac{x_{q}^{+}+x_{q}^{-}}{2}, y<\frac{y_{q}^{+}+y_{q}^{-}}{2}, \text { and } z<\frac{z_{q}^{+}+z_{q}^{-}}{2}\right\}, \\
& \Sigma^{-}=\left\{(x, y, z) \in \mathbb{R}^{3} \mid x<x_{q}^{-} \text {and } y=y_{q}^{-}+\rho_{1} \text {, with } \rho_{1}>0 \text { small }\right\}, \\
& \Sigma^{+}=\left\{(x, y, z) \in \mathbb{R}^{3} \mid x>x_{q}^{-} \text {and } y=y_{q}^{+}-\rho_{2}, \text { with } \rho_{2}>0 \text { small }\right\},
\end{aligned}
$$

as well as the corresponding transition maps,

$$
\begin{gathered}
\pi_{\text {out }}^{-}: \Sigma^{-} \rightarrow \Delta^{-}, \quad \pi_{\text {in }}^{+}: \Delta^{-} \rightarrow \Sigma^{+}, \quad \pi_{\text {out }}^{+}: \Sigma^{+} \rightarrow \Delta^{-}, \quad \text { and } \\
\pi_{\text {in }}^{-}: \Delta^{+} \rightarrow \Sigma^{-},
\end{gathered}
$$

that are induced by the flow of (2) for sufficiently small $\mu \in M$ and $\varepsilon, \delta>0$ (see Fig. 7). We hence need to prove that the return map $\pi=\pi_{\text {in }}^{-} \circ \pi_{\text {out }}^{+} \circ \pi_{\text {in }}^{+} \circ \pi_{\text {out }}^{-}: \Sigma^{-} \rightarrow \Sigma^{-}$is well-defined.

We first consider the case where the folded singularities $q^{-}$ and $q^{+}$are connected. Then, the maps $\pi_{\text {in }}^{\mp}$ are well-defined by standard GSPT; in particular, the constants $\rho_{1}$ and $\rho_{2}$ are sufficiently small such that trajectories with initial conditions on $\left.\Delta^{+}\right|_{\left\{z>z_{q}^{+}\right\}}$, respectively, $\left.\Delta^{-}\right|_{\left\{z<z_{q}^{-}\right\}}$, are attracted exponentially close to $\mathcal{Z}_{\varepsilon \delta}^{-}$, respectively, $\mathcal{Z}_{\varepsilon \delta}^{+}$, before crossing $\Sigma^{-}$, respectively, $\Sigma^{+}$. The welldefinedness of $\pi_{\text {out }}^{\mp}$ follows from Ref. 23, where it was shown that trajectories which approach the vicinities of $\mathcal{L}^{+}$and $\mathcal{L}^{-}$exponentially close to $\mathcal{Z}_{\varepsilon \delta}^{+}$and $\mathcal{Z}_{\varepsilon \delta}^{-}$, respectively, diverge exponentially from the latter at most at a buffer point that is bounded between $q^{+}$and $p^{+}$, respectively, between $q^{-}$and $p^{-}$.

We hence conclude that the map $\pi: \Sigma^{-} \rightarrow \Sigma^{-}$is welldefined for all sufficiently small $\varepsilon, \delta>0$. It follows that orbits of (2) are attracted to either $\mathcal{Z}_{\varepsilon \delta}^{-}$or $\mathcal{Z}_{\varepsilon \delta}^{+}$irrespective of initial condition, possibly after a jump; then, they follow the slow flow until they have to jump and are attracted to either $\mathcal{Z}_{\varepsilon \delta}^{-}$or $\mathcal{Z}_{\varepsilon \delta}^{+}$, which shows the existence of an attractor with double epochs of perturbed slow dynamics, as claimed.

Finally, the above argument also holds for the case of aligned singularities: while the folded singularities $q^{\mp}$ have the same $z$-coordinates in the singular limit of $\varepsilon=0=\delta$, the corresponding buffer points in the perturbed Eq. (2), with $\varepsilon, \delta>0$ being sufficiently small, are still bounded between $q^{\mp}$ and $p^{\mp}$, respectively. Hence, orbits jump at points near $\mathcal{L}^{ \pm}$and then cross $\Delta^{ \pm}$and are, therefore, attracted to both $\mathcal{Z}_{\varepsilon \delta}^{-}$and $\mathcal{Z}_{\varepsilon \delta}^{+}$, as in the connected case.

We remark that Theorem 1 guarantees the existence of an attractor for Eq. (2) with double epochs of perturbed slow dynamics; however, a more specific characterization, such as of its periodicity or chaoticity, is dependent on the properties of the function $\phi(x, y, z)$ in (2c) and hence requires a case-by-case study. Moreover, we note that the "double epoch" regime can be further divided into subregimes where SAOs occur "above" and "below"; SAOs are seen "above" with SAO-less slow dynamics below, or vice versa; or "threetimescale" relaxation oscillation is found, with the flow alternating between fast, intermediate, and slow SAO-less dynamics. Again, a precise characterization requires careful consideration of the given function $\phi(x, y, z)$ in (2c) [thus, for instance, we typically observe double-epoch MMO trajectories with SAOs only before relaxation 
in the Koper model from chemical kinetics studied in Sec. IV (cf. Figs. 1 and 11)].

\section{B. Remote singularities}

In the case where the folded singularities of $\mathcal{M}_{1}$ in (2) are remote, recall Definition 2 and Fig. 5, the perturbed flow of Eq. (2) with $\varepsilon, \delta>0$ being sufficiently small can exhibit MMOs with single epochs of SAOs, or "two-timescale" relaxation oscillation where the flow alternates between the fast and the intermediate dynamics for $\mu \in M$ (see again Fig. 6 and (32)).

First, to show the existence of relaxation oscillation in a $\mu$-subinterval of $M$, we combine the approaches of Refs. 18 and 30. To leading order in $\varepsilon$ and $\delta$, the $\mu$-values, which separate the corresponding parameter regimes can be determined by requiring that the intermediate flow on $\mathcal{S}^{a^{-}}$is "balanced" by that on $\mathcal{S}^{a^{+}}$. To that end, we consider the singular limit of $\varepsilon=0$ with $\delta>0$ being sufficiently small in (2); in other words, we restrict to the flow on $\mathcal{S}^{a^{\mp}}$, neglecting the $z$-displacement due to the fast flow (in $\varepsilon$ ) away from $\mathcal{S}^{a^{\mp}}$ (see Ref. 18 for details). We begin by defining

$$
x_{0}:=-\frac{f_{2}}{f_{3}}, \quad x_{\max }:=-\frac{2 f_{2}}{3 f_{3}}, \quad \text { and } \quad x_{\max }^{*}:=\frac{f_{2}}{3 f_{3}},
$$

where $x_{0}$ is the $x$-coordinate of $P\left(\mathcal{L}^{-}\right), x_{\text {max }}^{*}$ is the $x$-coordinate of $P\left(\mathcal{L}^{+}\right)$, and $x_{\max }$ is the $x$-coordinate of $\mathcal{L}^{+}$(cf. Fig. 8); here, we recall that $P\left(\mathcal{L}^{\mp}\right) \subset \mathcal{S}^{a^{ \pm}}$denotes the projection of $\mathcal{L}^{\mp}$ onto $\mathcal{S}^{a^{ \pm}}$along the fast fibers of (8a).

We then define

$$
\mathcal{G}_{0}^{-}(z, \mu):=\mathcal{G}\left(x_{\max }^{*}, 0 ; z ; \mu\right), \quad \mathcal{G}_{0}^{+}(z, \mu):=\mathcal{G}\left(x_{0}, x_{\max } ; z ; \mu\right),
$$

$$
\mathcal{R}(z, \mu):=\mathcal{G}_{0}^{-}(z, \mu)+\mathcal{G}_{0}^{+}(z, \mu),
$$

where we recall that

$$
\mathcal{G}\left(x_{0}, x_{1} ; z_{0} ; \mu\right)=\int_{x_{0}}^{x_{1}} \frac{F^{\prime}(\sigma)\left(\mu+\phi\left(\sigma, F(\sigma), z_{0}\right)\right)}{\alpha \sigma+\beta F(\sigma)-z_{0}} \mathrm{~d} \sigma
$$

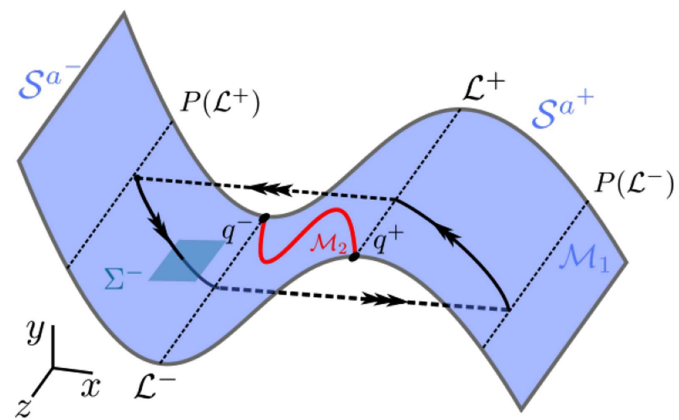

(a) is obtained by eliminating time in the reduced flow on $\mathcal{S}^{a^{\mp}}$ under (17) and integrating (cf. Lemma 1). Finally, for future reference, we also write

$$
I:=\left(z_{q}^{-}, z_{q}^{+}\right) \quad \text { and } \quad \bar{I}:=\left[z_{q}^{-}, z_{q}^{+}\right] .
$$

To leading order in $\delta$ and for appropriately restricted $\mu$-values, as specified below, the singular (in $\varepsilon$ ) trajectory through a point $\left.(0,0, z) \in \mathcal{L}^{-}\right|_{I}$ returns to a point $(0,0, \hat{z})$ on $\left.\mathcal{L}^{-}\right|_{I}$, where

$$
\hat{z}=z+\delta \mathcal{R}(z, \mu)+\mathcal{O}\left(\delta^{2}\right) .
$$

In the following, we will show that this map is well-defined (see Theorem 2 below).

We will say that the flow on $\mathcal{S}^{a^{-}}$is balanced by that on $\mathcal{S}^{a^{+}}$at a point with $z=z^{*}$ for $\delta>0$ being sufficiently small if $\hat{z}=z^{*}$, i.e., if $\mathcal{R}\left(z^{*}, \mu\right)=0$. We will require the following technical result.

Lemma 2. If the folded singularities $q^{\mp}$ of $\mathcal{M}_{1}$ are remote, i.e., if $\frac{\alpha}{\beta}<\frac{2 f_{2}^{2}}{9 f_{3}}$, then

$$
\begin{gathered}
\int_{x_{0}}^{x_{\max }} \frac{F^{\prime}(\sigma)}{\alpha \sigma+\beta F(\sigma)-z_{q}^{\mp}} \mathrm{d} \sigma>0 \text { and } \\
\int_{x_{\max }^{*}}^{0} \frac{F^{\prime}(\sigma)}{\alpha \sigma+\beta F(\sigma)-z_{q}^{\mp}} \mathrm{d} \sigma>0,
\end{gathered}
$$

as well as

$$
\mathcal{G}_{0}^{-}\left(z_{q}^{-}, \mu\right)>0 \text { and } \mathcal{G}_{0}^{+}\left(z_{q}^{+}, \mu\right)<0
$$

for $\mu \in \bar{M}$.

Proof. The assertions in (38) and (39) follow immediately from the below:

1. $F^{\prime}(x)<0$ for $x \in\left(x_{\max }^{*}, 0\right) \cup\left(x_{0}, x_{\max }\right)$, i.e., on $\mathcal{S}^{a}$, recall Eq. (13);

2. $\alpha x+\beta F(x)-z<0$ for $(x, z) \in\left(x_{\max }^{*}, 0\right) \times \bar{I}$, recall Eq. (24) and Fig. 5(a);

3. $\alpha x+\beta F(x)-z>0$ for $(x, z) \in\left(x_{0}, x_{\max }\right) \times \bar{I}$, again by (24) and Fig. 5(a);

4. $\mu+\phi(\sigma, F(\sigma), z)>0$ for $(x, z, \mu) \in\left(x_{\max }^{*}, 0\right) \times \bar{I} \times \bar{M}$, recall Assumption 2; and

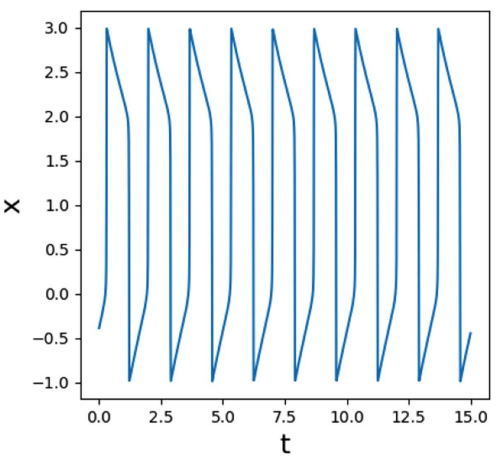

(b)

FIG. 8. Schematic illustration of the emergence of relaxation oscillation in (2) for the case of remote singularities: (a) singular geometry and (b) corresponding time series for the Koper model studied in Sec. IV. 
5. $\mu+\phi(\sigma, F(\sigma), z)<0$ for $(x, z, \mu) \in\left(x_{0}, x_{\max }\right) \times \bar{I} \times \bar{M}$, again by Assumption 2 .

The values $\mu_{r}^{-}$and $\mu_{r}^{+}$for which the reduced flow on $\mathcal{S}^{a^{-}}$is balanced by that on $\mathcal{S}^{a^{+}}$at $q^{-}$and $q^{+}$, respectively, to leading order in $\delta>0$ are found by solving $\mathcal{R}\left(z_{q}^{-}, \mu\right)=0$ and $\mathcal{R}\left(z_{q}^{+}, \mu\right)=0$, respectively. The relative position of $\mu_{r}^{-}$and $\mu_{r}^{+}$on the real line depends on the properties of the function $\phi(x, y, z)$ in (2c); we, therefore, make the following assumption.

Assumption 3. Denote by $\mu_{r}^{\mp}$ the $\mu$-values for which

$$
\mathcal{R}\left(z_{q}^{\mp}, \mu_{r}^{\mp}\right)=0
$$

holds, i.e., define

$$
\mu_{r}^{\mp}:=-\frac{\int_{x_{\max }^{*}}^{0} \frac{F^{\prime}(\sigma) \phi\left(\sigma, F(\sigma), z_{q}^{\mp}\right)}{\alpha \sigma+\beta F(\sigma)-z_{q}^{\mp}} \mathrm{d} \sigma+\int_{x_{0}}^{x_{\max }} \frac{\frac{F^{\prime}(\sigma) \phi\left(\sigma, F(\sigma), z_{q}^{\mp}\right)}{\alpha \sigma+\beta F(\sigma)-z_{q}^{\mp}}}{\alpha} \mathrm{d} \sigma}{\int_{x_{\max }^{*}}^{0} \frac{F^{\prime}(\sigma)}{\alpha \sigma+\beta F(\sigma)-z_{q}^{\mp}} \mathrm{d} \sigma+\int_{x_{0}}^{x_{\max }} \frac{F^{\prime}(\sigma)}{\alpha \sigma+\beta F(\sigma)-z_{q}^{\mp}} \mathrm{d} \sigma} .
$$

Then, we assume that

$$
\mu_{q}^{-}<\mu_{r}^{-}<\mu_{r}^{+}<\mu_{q}^{+} .
$$

Clearly, Lemma 2 now implies that the denominator in (41) is non-zero. Assumption 3 is essentially an assumption on the properties of the function $\phi(x, y, z)$ in (2c), which is made for consistency with the Koper model [Eq. (1)] after transformation to the prototypical Eq. (2). We now make an additional assumption on $\phi(x, y, z)$, which is also consistent with the Koper model.

Assumption 4. We assume that $\partial_{z} \phi(x, y, z) \leq 0$ for $z \in \bar{I}$.

Here and in the following, we denote partial derivatives by $\partial_{\square}$, for brevity of notation. We remark that Assumption 4 is sufficient, but not necessary, for the occurrence of relaxation oscillation in (2) (see also the discussion following Theorem 2 below). We now introduce a final preliminary technical result.

Lemma 3. Assume that Assumption 4 holds and that the folded singularities of Eq. (2) are remote in the sense of Definition 2, i.e., that $\frac{\alpha}{\beta}<\frac{2 f_{2}^{2}}{9 f_{3}}$ in (2). Then, the following holds for $z \in \bar{I}$ $\times\left[\mu_{r}^{-}, \mu_{r}^{+}\right]$:

$$
\partial_{\mu} \mathcal{R}(z, \mu)<0
$$

and

$$
\partial_{z} \mathcal{R}(z, \mu)>0 .
$$

Proof. The proof is similar to that of Lemma 2.

We now state our main result in this section:

Theorem 2. Assume that Assumption 1 through Assumption 4 hold, that the folded singularities of Eq. (2) are remote in the sense of Definition 2, i.e., that $\frac{\alpha}{\beta}<\frac{2 f_{2}^{2}}{9 f_{3}}$ in (2), and fix $\mu \in\left(\mu_{r}^{-}, \mu_{r}^{+}\right)$. Then, there exist sufficiently small $\varepsilon_{0}, \delta_{0}>0$ such that Eq. (2) admits a stable relaxation oscillation orbit for all $(\varepsilon, \delta) \in\left(0, \varepsilon_{0}\right) \times\left(0, \delta_{0}\right)$.

Proof. The proof in based on showing that the assumptions of Theorem 4 of Ref. 30 are satisfied.

- Assumption 1 in Theorem 4 of Ref. 30. By construction, the manifold $\mathcal{M}_{1}$ is $S$-shaped, with two attracting sheets $\mathcal{S}^{a^{\mp}}$ separated by a repelling sheet $\mathcal{S}^{r}$ [recall (12), (13), and (14)].
- Assumption 2 in Theorem 4 of Ref. 30. The transversality condition is satisfied on $\left.\mathcal{L}^{\mp}\right|_{I}$.

- Assumptions 3 and 4 in Theorem 4 of Ref. 30. In the singular limit of $\varepsilon=0=\delta$, one can construct a one-dimensional map,

$$
\sigma^{-}:\left.P\left(\mathcal{L}^{+}\right)\right|_{I} \rightarrow \mathcal{L}^{-}, \quad z \mapsto z,
$$

where we recall that $P: \mathcal{L}^{\mp} \rightarrow \mathcal{S}^{a^{ \pm}}$is the projection of a point on $\mathcal{L}^{\mp}$ to a point on $\mathcal{S}^{a^{ \pm}}$along the fast fibers of (8a). For sufficiently small $\delta>0$, the map $\sigma^{-}$perturbs smoothly to

$$
\sigma_{\delta}^{-}:\left.P\left(\mathcal{L}^{+}\right)\right|_{I} \rightarrow \mathcal{L}^{-}, \quad z \mapsto z+\mathcal{O}(\delta) .
$$

In particular, by eliminating time in (17), we may approximate the map $\sigma_{\delta}^{-}$by

$$
z \mapsto z+\delta \mathcal{G}_{0}^{-}(z, \mu)+\mathcal{O}\left(\delta^{2}\right) .
$$

Moreover, since

$$
\mathcal{G}_{0}^{-}\left(z_{q}^{-}, \mu\right)>0
$$

for $\mu \in M$, by Lemma 3, the map $\sigma_{\delta}^{-}:\left.\left.P\left(\mathcal{L}^{+}\right)\right|_{I} \rightarrow \mathcal{L}^{-}\right|_{\left\{z>z_{q}^{-}\right\}}$is well-defined, with $\sigma_{\delta}^{-}(z) \rightarrow \sigma^{-}(z)$ as $\delta \rightarrow 0$ uniformly in $z$. In particular, (47) implies that the point on $P\left(\mathcal{L}^{+}\right)$with $z=z_{q}^{-}$is mapped to a point on $\mathcal{L}^{-}$with $z>z_{q}^{-}$. Since the map $\sigma_{\delta}^{-}$is induced by the reduced flow on $\mathcal{S}^{a^{-}}$, it follows by existence and uniqueness of solutions that all points on $P\left(\mathcal{L}^{+}\right)$with $z \geq z_{q}^{-}$are mapped to points on $\mathcal{L}^{-}$with $z>z_{q}^{-}$. Similarly, one can construct a map $\sigma_{\delta}^{+}:\left.\left.P\left(\mathcal{L}^{-}\right)\right|_{I} \rightarrow \mathcal{L}^{+}\right|_{\left\{z<z_{q}^{+}\right\}}$, under which points on $P\left(\mathcal{L}^{-}\right)$with $z \leq z_{q}^{+}$are mapped to points on $\mathcal{L}^{+}$with $z<z_{q}^{+}$. The composition of $\sigma_{\delta}^{-}$and $\sigma_{\delta}^{+}$with $P$ defines the return map,

$\pi^{-}:=\sigma_{\delta}^{-} \circ P \circ \sigma_{\delta}^{+} \circ P:\left.\left.\mathcal{L}^{-}\right|_{I} \rightarrow \mathcal{L}^{-}\right|_{I}, \quad z \mapsto z+\delta \mathcal{R}(z, \mu)$.

Since

$$
\partial_{\mu} \mathcal{R}(z, \mu)>0 \quad \text { for all }(z, \mu) \in I \times\left(\mu_{r}^{-}, \mu_{r}^{+}\right),
$$

i.e., since $\mathcal{R}(z, \mu)$ is an increasing function of $\mu$ by Lemma 3 , and since, moreover,

$$
\mathcal{R}\left(z_{q}^{-}, \mu_{r}^{-}\right)=0 \text { and } \mathcal{R}\left(z_{q}^{+}, \mu_{r}^{+}\right)=0
$$

by Assumption 3, it correspondingly follows that

$$
\mathcal{R}\left(z_{q}^{-}, \mu\right)>0 \text { and } \mathcal{R}\left(z_{q}^{+}, \mu\right)<0 \text { for all } \mu \in\left(\mu_{r}^{-}, \mu_{r}^{+}\right),
$$

and the map $\pi^{-}:\left.\left.\mathcal{L}^{-}\right|_{I} \rightarrow \mathcal{L}^{-}\right|_{I}$ is, therefore, well-defined. By the intermediate value theorem, for any $\mu \in\left(\mu_{r}^{-}, \mu_{r}^{+}\right)$, there exists $z^{*} \in I$ such that $\mathcal{R}\left(z^{*}, \mu\right)=0$; therefore, $z^{*}$ is a fixed point of $\pi^{-}$. Moreover, from (42), it follows that $\partial_{z} \mathcal{R}(z, \mu)<0$ for all $(z, \mu) \in \bar{I} \times\left[\mu_{r}^{-}, \mu_{r}^{+}\right]$; therefore, the fixed point $z^{*} \in I$ is unique, which implies the existence of a unique singular cycle for any $\delta>0$ sufficiently small.

- Assumption 5 in Theorem 4 of Ref. 30. Consider the section

$$
\Sigma^{-}=\left\{(x, y, z) \in \mathbb{R}^{3} \mid x<x_{q}^{-} \text {and } y=y_{q}^{-}+\rho_{1} \text {, with } \rho_{1}>0\right\}
$$

(see Fig. 8). The return map $\pi^{-}: \mathcal{L}^{-} \rightarrow \mathcal{L}^{-}$in (48) induces a return map $\Pi^{-}: \Sigma^{-} \rightarrow \Sigma^{-}$. Correspondingly, fixed points of 
$\pi^{-}$on $\mathcal{L}^{-}$are connected to fixed points of $\Pi^{-}$on $\Sigma^{-}$via trajectories of the reduced flow in (17) on $\mathcal{S}^{a^{-}}$. In particular, a fixed point of $\Pi^{-}$is hyperbolic and attracting, respectively, repelling, if and only if the corresponding fixed point of $\pi^{-}$is hyperbolic and attracting, respectively, repelling. Since $\partial_{z}(z+\delta \mathcal{R}(z, \mu))<1$ for all $z \in \bar{I}$, by (42), $z^{*}$ is attracting in our case, as is the corresponding fixed point of $\Pi^{-}$on $\Sigma^{-}$, which shows the existence of a hyperbolic and attracting singular periodic orbit.

The above assumptions are established in the singular limit of $\varepsilon=0$, with $\delta>0$ being sufficiently small. For $\varepsilon, \delta>0$ small and $z \in I$, a trajectory with initial condition $\left(x_{\max }^{*}, y_{q}^{+}, z\right)$ on $\mathcal{S}_{\varepsilon \delta}^{a^{-}}$-i.e., at the height of $P\left(\mathcal{L}^{+}\right)$-is mapped to a point on $\mathcal{S}_{\varepsilon \delta}^{a^{-}}$under the flow of (2) and following a large excursion:

$$
z \mapsto z+\delta \mathcal{R}(z, \mu)+\mathcal{O}\left(\delta^{2}, \delta \varepsilon \ln \varepsilon\right) .
$$

The above map is smooth in $z$ and $\mu$. To see that (49) holds, we note that in the double singular limit of $\varepsilon=0=\delta$, the return map is the identity which, for $\varepsilon=0$ and $\delta>0$ small, then perturbs to (48). By Theorems 3 and 4 of Ref. 30, an $\mathcal{O}(\varepsilon \ln \varepsilon)$-contribution arises through the perturbation of the functions $\mathcal{G}_{0}^{\mp}$ for $\varepsilon, \delta>0$ being sufficiently small, which is multiplied by a factor of $\delta$ in our case since the $z$-displacement needs to be zero for $\delta=0$ regardless of $\varepsilon$, as can be seen by setting $\delta=0$ in (6). Hence, (49) follows.

We reiterate that Assumption 4 is sufficient, but not necessary for stable relaxation oscillation to occur, as described in Theorem 2. Specifically, Lemma 3 guarantees the existence and uniqueness of a singular cycle in the limit of $\varepsilon=0$, with $\delta>0$ small (see the third bullet point in the proof of Theorem 2). In general, the function $\phi(x, y, z)$ could be such that more than one singular cycle exists in that limit. The stability of each of those would be studied individually, which would result in the existence of more than one relaxation oscillation orbit for $\varepsilon, \delta>0$ small, some of which could potentially be stable, while others could be unstable. We reiterate that Assumption 4 is satisfied for the Koper model from chemical kinetics, which is studied in Sec. IV.

We now state our main result on the existence of MMOs with single epochs of perturbed slow dynamics.

Theorem 3. Assume that Assumption 1 through Assumption 4 hold, that the folded singularities of Eq. (2) are remote in the sense of Definition 2, i.e., that $\frac{\alpha}{\beta}<\frac{2 f_{2}^{2}}{9 f_{3}}$ in (2), and fix $\mu \in\left(\mu_{q}^{-}, \mu_{r}^{-}\right)$. Then, there exist sufficiently small $\varepsilon_{0}, \delta_{0}>0$ such that Eq. (2) features MMOs with single epochs of perturbed slow dynamics "below" for all $(\varepsilon, \delta) \in\left(0, \varepsilon_{0}\right) \times\left(0, \delta_{0}\right)$.

Proof. Consider the section

$$
\Sigma^{-}=\left\{(x, y, z) \in \mathbb{R}^{3} \mid x<x_{q}^{-} \text {and } y=y_{q}^{-}+\rho_{1} \text {, with } \rho_{1}>0 \text { small }\right\} \text {. }
$$

As in Theorems 1 and 2, the return map $\Pi^{-}: \Sigma^{-} \rightarrow \Sigma^{-}$induced by the flow of (2) is well-defined for sufficiently small $\mu \in\left(\mu_{q}^{-}, \mu_{r}^{-}\right)$ and $\varepsilon, \delta>0$

Consider now a point $\left(x_{\max }^{*}, y_{q}^{+}, z\right)$ on $\mathcal{S}_{\varepsilon \delta}^{a^{-}}$, i.e., at the height of $P\left(\mathcal{L}^{+}\right)$, with $z \in I$. The corresponding trajectory returns to $\mathcal{S}_{\varepsilon \delta}^{a^{-}}$, after a large excursion, at a point with $z+\delta \mathcal{R}(z, \mu)+\mathcal{O}\left(\delta^{2}, \delta \varepsilon \ln \varepsilon\right)$ (recall the proof of Theorem 2). Moreover, there holds that $\mathcal{R}(z, \mu)<0$ for all $(z, \mu) \in I \times\left(\mu_{q}^{-}, \mu_{r}^{-}\right)$, which follows from (42) and (43). Therefore, the trajectory "drifts" toward the negative $z$-direction until it reaches a point $\left(x_{\max }^{*}, y_{q}^{+}, z\right)$ on $\mathcal{S}_{\varepsilon \delta}^{a^{-}}$with $z<z_{q}^{-}$, i.e., until it enters the funnel of $q^{-}$. We reiterate that, in a first approximation, the funnel of $q^{-}$is the area in $\mathcal{S}^{a^{-}}$bounded by $\mathcal{Z}^{-}$ and the intermediate fiber of (21) that crosses $q^{-}$, which is given by $\left\{z=z_{q}^{-}\right\}$.

Points $\left(x_{\max }^{*}, y_{q}^{+}, z\right)$ on $\mathcal{S}_{\varepsilon \delta}^{a^{-}}$with $z<z_{q}^{-}$are attracted to the vicinity of $q^{-}$and undergo SAOs. According to Refs. 13 and 21, the buffer point beyond which all trajectories have to diverge exponentially from $\mathcal{Z}^{-}$lies $o(1)$-close to $q^{-}$. Therefore, there exist sufficiently small $\varepsilon_{0}, \delta_{0}>0$, which satisfy, in particular, $\varepsilon_{0}<\left(z_{q}^{+}-z_{q}^{-}\right)^{2}$ and $\delta_{0}<z_{q}^{+}-z_{q}^{-}$such that for all $(\varepsilon, \delta) \in\left(0, \varepsilon_{0}\right) \times\left(0, \delta_{0}\right)$, trajectories that diverge exponentially from $\mathcal{Z}_{\varepsilon \delta}^{-}$undergo a slow drift toward the negative $z$-direction, without interacting with $\mathcal{Z}_{\varepsilon \delta}^{+}$, until they enter the funnel of $q^{-}$. The above implies the existence of MMO trajectories with single epochs of perturbed slow dynamics, as claimed. $\square$

Remark 4. Without loss of generality, we restrict to the case where perturbed slow dynamics occurs "below" in Theorem 3; the occurrence "above," for $\mu \in\left(\mu_{r}^{+}, \mu_{q}^{+}\right)$, can be shown in an analogous fashion.

The requirement that $\varepsilon_{0}<\left(z_{q}^{+}-z_{q}^{-}\right)^{2}$ and $\delta_{0}<z_{q}^{+}-z_{q}^{-}$is a sufficient condition which guarantees that MMOs with single epochs of perturbed slow dynamics exist, regardless of how close the folded singularities are in the $z$-direction. As will become apparent in Subsection 1 in the Appendix, the buffer point near $\mathcal{L}^{-}$ lies $\mathcal{O}(\sqrt{\varepsilon}, \delta)$-close to the fold line $\mathcal{L}^{-}$. Hence, trajectories which diverge exponentially from $\mathcal{Z}^{-}$are not able to reach $\mathcal{Z}^{+}$. If the singularities $q^{\mp}$ are remote but sufficiently close in the $z$-direction, then trajectories can potentially interact with both $\mathcal{Z}^{\mp}$ for (sufficiently large) values of $\varepsilon$ and $\delta$; correspondingly, Eq. (2) can feature MMOs with double epochs of perturbed slow dynamics, or even more exotic patterns where the two epochs are separated by LAOs, in that case; an example of the latter in the context of the multi-timescale Hodgkin-Huxley equations from mathematical neuroscience is included in the upcoming work, Ref.16. We remark that we have not been able to find such "exotic" behavior in the context of the Koper model from chemical kinetics (cf. Sec. IV).

Finally, with regard to the number of LAOs that can occur between SAO segments, we have the following result.

Corollary 1. Assume that Assumption 1 through Assumption 4 hold, that the folded singularities of Eq. (2) are remote in the sense of Definition 2, i.e., that $\frac{\alpha}{\beta}<\frac{2 f_{2}^{2}}{9 f_{3}}$ in (2), fix $\mu \in\left(\mu_{q}^{-}, \mu_{r}^{-}\right)$and consider $\varepsilon, \delta>0$ sufficiently small. Denote by $p_{\text {out }}=\left(x_{\text {out }}, y_{\text {out }}, z_{\text {out }}\right)$ the point at which a given trajectory diverges exponentially from $\mathcal{Z}_{\varepsilon \delta}^{-}$, with $z_{\text {out }}>0$, and denote by $L$ the number of large excursions that follow before the trajectory is again attracted exponentially close to $\mathcal{Z}_{\varepsilon \delta}^{-}$. Then, the following holds:

1. If $z_{\text {out }}+\delta \mathcal{R}(0, \mu)<0$, then $L=1$;

2. if $0<z_{\text {out }}+\delta \mathcal{R}(0, \mu)<z_{\text {out }}$, then

$$
L=1+\left\lfloor\frac{z_{\text {out }}}{\delta \mathcal{R}(0, \mu)}\right\rfloor
$$


where \lfloor\rfloor denotes the floor function.

Proof. Both statements follow immediately from Theorem 3.

\section{Summary}

In summary, the emergence of mixed-mode dynamics in (2) can, thus, be understood as follows: By standard GSPT, ${ }^{10}$ the normally hyperbolic portions $\mathcal{S}^{a^{\mp}}$ and $\mathcal{Z}^{\mp}$ of $\mathcal{M}_{1}$ and $\mathcal{M}_{2}$, respectively, perturb to $\mathcal{S}_{\varepsilon \delta}^{a^{\mp}}$ and $\mathcal{Z}_{\varepsilon \delta}^{\mp}$, respectively. Given an initial point $(x, y, z)$ $\in \mathcal{S}_{\varepsilon \delta}^{a^{-}}$, the corresponding trajectory will follow the intermediate flow on $\mathcal{S}_{\varepsilon \delta}^{a^{-}}$until it is either attracted to $\mathcal{Z}_{\varepsilon \delta}^{-}$or until it reaches the vicinity of $\mathcal{L}^{-}$. In the former case, the trajectory then follows the slow flow on $\mathcal{Z}_{\varepsilon \delta}^{-}$and can undergo SAOs; in the latter case, no slow dynamics occurs, and the trajectory jumps near $\mathcal{L}^{-}$to the opposite attracting sheet $\mathcal{S}_{\varepsilon \delta}^{a^{+}}$, resulting in a large excursion. The above sequence then begins anew (see Figs. 7-9 for schematic illustrations): depending on the relative geometry of the folded singularities $q^{\mp}$ of $\mathcal{M}_{1}$, oscillatory trajectories with single, double, or no epochs of slow dynamics can occur, as indicated in Fig. 6.

We emphasize that the "double epoch" regime in panel (b) of Fig. 6 does not necessarily imply mixed-mode dynamics with two epochs of SAOs but, rather, with double epochs of perturbed slow dynamics of the corresponding singular cycles. That is, MMO trajectories are attracted to the vicinity of both branches $\mathcal{Z}_{\varepsilon \delta}^{\mp}$ and, hence, exhibit slow dynamics; however, whether SAOs will occur depends on which region on $\mathcal{Z}$ trajectories enter: by Lemma 4 in the Appendix, they may experience either focal or nodal attraction. In particular, if a trajectory is attracted to the focal region on both $\mathcal{Z}_{\varepsilon \delta}^{-}$and $\mathcal{Z}_{\varepsilon \delta}^{+}$, then two epochs of SAOs are observed. On the other hand, trajectories that are first attracted to the focal region on, say, $\mathcal{Z}^{-}$before being attracted to and repelled from the nodal region on $\mathcal{Z}^{+}$feature SAOs below and mere slow dynamics above (the corresponding segment of the associated Farey sequence would be $1_{s} 1^{0}$, with $s>0$ ). Similarly, a trajectory that is attracted to and repelled from nodal regions on both $\mathcal{Z}^{-}$and $\mathcal{Z}^{+}$features no SAOs at all and is hence a relaxation oscillation with fast, intermediate, and slow components; the associated Farey sequence would be $1_{0} 1^{0}$. In the transition between remote and connected singularities, exotic MMO trajectories may occur which contain segments of two-timescale relaxation oscillation, SAOs below, and SAOs above (the associated Farey sequence would be $L_{k} 1^{s}$, with $L, k, s>0$ ). Moreover, we postulate that chaotic mixed-mode dynamics may be possible. However, the above characterization depends substantially on the particular form of the function $\phi$ in (2c); it is, hence, not feasible to further subdivide that region in Fig. 6 on the basis of system parameters alone. Rather, a case-by-case study is required.

Finally, we remark on the role of the ratio between the scale separation parameters $\varepsilon$ and $\delta$ for the dynamics of Eq. (2). Locally, in order for the system to exhibit three timescales and for the iterative reduction from the fast via the intermediate to the slow dynamics to be accurate, $\varepsilon$ and $\delta$ need to be sufficiently small, which is akin to asking "When is $\varepsilon$ small enough?" in a two-timescale system. The resulting three-timescale dynamics then features characteristics of both two-fast/one-slow systems and one-fast/two-slow systems; the resulting SAOs will be either of sector type or of delayed Hopf type. Moreover, the width of the corresponding regions on $\mathcal{Z}$ is either $\mathcal{O}(\varepsilon)$ or $\mathcal{O}(\sqrt{\varepsilon})$, respectively (see the Appendix for details). By Ref. 18 and Lemma 1, the "step" in the $z$-direction taken by trajectories after a large excursion and re-injection is $\mathcal{O}(\delta)$; it, therefore, follows that if $\delta=\mathcal{O}\left(\varepsilon^{c}\right)$ for $0<c<1$, then trajectories will typically not undergo sector-type dynamics since the width of the latter regime is $\mathcal{O}(\varepsilon)$. Hence, delay-type SAOs are expected to dominate in that case (see Fig. 12 below for an example). The above observations are in agreement with a similar conjecture made in Section 7.1 of Ref. 31.

\section{THE KOPER MODEL REVISITED}

In this section, we revisit the Koper model from chemical kinetics [Eq. (1)], which we reiterate to be a particular realization of Eq. (2) for

$$
\varepsilon=\frac{\epsilon}{|k|}, \quad f_{2}=\frac{3}{|k|}, \quad f_{3}=-\frac{1}{|k|},
$$

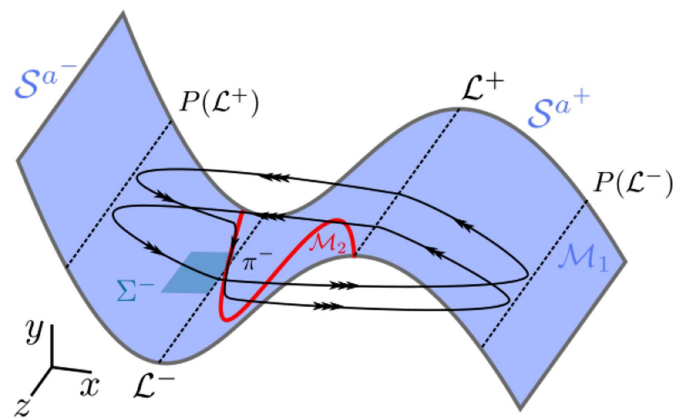

(a)

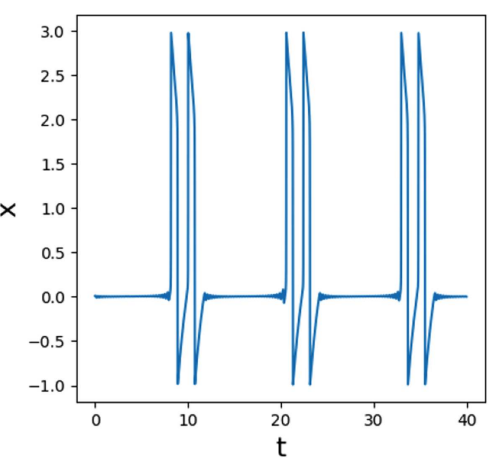

(b)

FIG. 9. Schematic illustration of the emergence of MMO trajectories with single epochs of perturbed slow dynamics in (2) for the case of remote singularities: (a) singular geometry and return map and (b) corresponding time series for the Koper model studied in Sec. IV. 


$$
\begin{gathered}
\alpha=1, \quad \beta=-2, \\
\mu=\frac{k+\lambda+2}{k}, \text { and } \phi(x, y, z)=-y-z,
\end{gathered}
$$

after the transformation $(x, y, z, \lambda, k, t) \rightarrow\left(x+1, y+\frac{2+\lambda}{|k|},-z-1\right.$ $\left.+\frac{2(2+\lambda)}{|k|}, \lambda, k, t\right)$. Henceforth, we will refer to (2) with the above choice of parameters as the Koper model; here, $k<0$ and $\lambda \in \mathbb{R}$ will be our bifurcation parameters.

From Sec. II, it is apparent that the effect of the parameter $k$ on the dynamics is more substantial than that of $\lambda$ since variation in $k$ simultaneously affects the timescale separation (through $\varepsilon$ ) and the singular geometry (through $f_{2}$ and $f_{3}$ ) as well as the slow flow and the global return (through $\mu$ ). Given $k<0$ fixed, on the other hand, the variation in $\lambda$ only affects the slow flow and the global return (through $\mu$ ). It is, therefore, the parameter $k$ that determines whether the folded singularities in the Koper model are remote, aligned, or connected, and whether the model can exhibit single or double epochs of SAOs. For given $k<0$, the parameter $\lambda$ can differentiate between steady-state and oscillatory behavior, as well as between mixed-mode dynamics and relaxation oscillation in the case of remote singularities, as will become clear in the following.

Remark 5. Alternatively, the Koper model can be written in the symmetric form

$$
\begin{gathered}
\epsilon \dot{x}=y-x^{3}+3 x, \\
\dot{y}=k x-2(y+\lambda)+z, \\
\dot{z}=\delta(\lambda+y-z),
\end{gathered}
$$

which is invariant under the transformation $(x, y, z, \lambda, k, t)$ $\rightarrow(-x,-y,-z,-\lambda, k, t)$.

In the following, we will restrict to the case where $\lambda>0$ in (1). Moreover, we will investigate the dynamics near $\mathcal{L}^{-}$only: by Remark 5 , the flow near $\mathcal{L}^{+}$for $\lambda<0$ can then be inferred by symmetry [cf. also panels (a) and (b) in Fig. 1], where the corresponding time series are seen to be symmetric about the $t$ axis for $k$ fixed and $\lambda \rightarrow-\lambda$.

\section{A. Singular geometry}

The critical and 2-critical manifolds $\mathcal{M}_{1}$ and $\mathcal{M}_{2}$, respectively, for the Koper model are given by

$$
\begin{gathered}
\mathcal{M}_{1}=\left\{(x, y, z) \in \mathbb{R}^{3} \mid y=x^{2} \frac{3-x}{|k|}\right\}, \\
\mathcal{M}_{2}=\left\{(x, y, z) \in \mathcal{M}_{1} \mid z=x-2 x^{2} \frac{3-x}{|k|}\right\}
\end{gathered}
$$

(see Sec. II). The normally hyperbolic portion $\mathcal{S}$ of the critical manifold $\mathcal{M}_{1}$ can be written as

$$
\mathcal{S}=\mathcal{S}^{a^{-}} \cup \mathcal{S}^{r} \cup \mathcal{S}^{a^{+}},
$$

where

$$
\begin{gathered}
\mathcal{S}^{a^{-}}=\left\{(x, y, z) \in \mathcal{M}_{1} \mid x<0\right\}, \\
\mathcal{S}^{r}=\left\{(x, y, z) \in \mathcal{M}_{1} \mid 0<x<2\right\}, \quad \text { and } \\
\mathcal{S}^{a^{+}}=\left\{(x, y, z) \in \mathcal{M}_{1} \mid x>2\right\} .
\end{gathered}
$$

The fold lines of $\mathcal{M}_{1}$ are located at

$$
\begin{gathered}
\mathcal{L}^{-}=\left\{(x, y, z) \in \mathbb{R}^{3} \mid x=0=y\right\} \quad \text { and } \\
\mathcal{L}^{+}=\left\{(x, y, z) \in \mathbb{R}^{3} \mid x=2 \text { and } y=\frac{4}{|k|}\right\} .
\end{gathered}
$$

The corresponding folded singularities $q^{\mp}$ are found at

$$
q^{-}=(0,0,0) \quad \text { and } \quad q^{+}=\left(2, \frac{4}{|k|}, 2-\frac{8}{|k|}\right) .
$$

We have the following result on the relative position of the singularities $q^{\mp}$ :

Proposition 5. Let $\varepsilon=0=\delta$. Then, the folded singularities of the Koper model are aligned for $k=-4$, connected when $-4<k$ $<0$, and remote for $k<-4$.

Proof. The statement follows from Proposition 3 and (3), or by comparison of the $z$-coordinates of $q^{-}$and $q^{+}$.

The 2-critical manifold $\mathcal{M}_{2}$ is normally hyperbolic everywhere except at the fold points $p^{\mp}$, where

$$
\begin{gathered}
x_{p}^{\mp}=1 \pm \sqrt{1-\frac{|k|}{6}}, \quad y_{p}^{\mp}=\frac{\left(2 \pm \sqrt{1-\frac{|k|}{6}}\right)\left(1 \mp \sqrt{1-\frac{|k|}{6}}\right)^{2}}{|k|}, \quad \text { and } \\
z_{p}^{\mp}=1 \mp \sqrt{1-\frac{|k|}{6}}-2 \frac{\left(2 \pm \sqrt{1-\frac{|k|}{6}}\right)\left(1 \mp \sqrt{1-\frac{|k|}{6}}\right)^{2}}{|k|} .
\end{gathered}
$$

Based on the above, we have the following:

Proposition 6. If $-6<k<0$, then $\mathcal{M}_{2}$ admits two fold points which are located between the points of intersection of $\mathcal{M}_{2}$ with $\mathcal{L}^{\mp}$, i.e., on the repelling sheet of $\mathcal{M}_{1}$. If $k<-6$, then $\mathcal{M}_{2}$ admits no fold points.

We reiterate that, due to $k<0$, the fold points $p^{\mp}$ in the Koper model cannot cross $\mathcal{L}^{\mp}$ and that the corresponding singular geometry is, therefore, as depicted in Fig. 4(c).

Remark 6. In Example 4.3 of Ref. 1, the manifold $\mathcal{M}_{2}$ is characterized as normally hyperbolic everywhere, in spite of its graph being $S$-shaped. Proposition 6 above shows that $\mathcal{M}_{2}$ can, in fact, admit two fold points at which normal hyperbolicity is lost.

\section{B. Classification of three-timescale dynamics}

Here, we classify the dynamics of the Koper model in the threetimescale context for various choices of the parameters $k$ and $\lambda$ in Eq. (1). In particular, we hence construct the two-parameter bifurcation diagram shown in Fig. 10 (recall Fig. 2) [a two-timescale analog of Fig. 10 for the case of one fast and two slow variables in (1) is presented in Ref. 6]. Given the definition of $\mu$ in (3), we consider $\lambda$ as a function of $k$ here when retracing the analysis from 


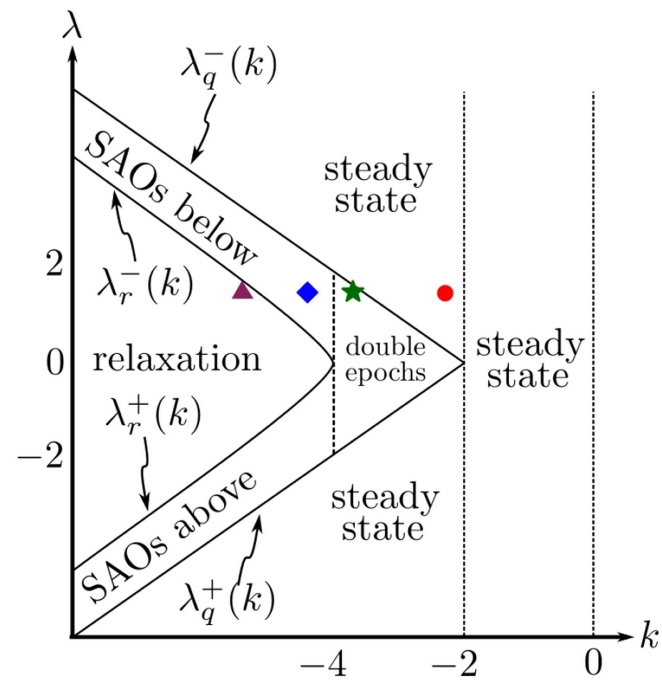

(a)

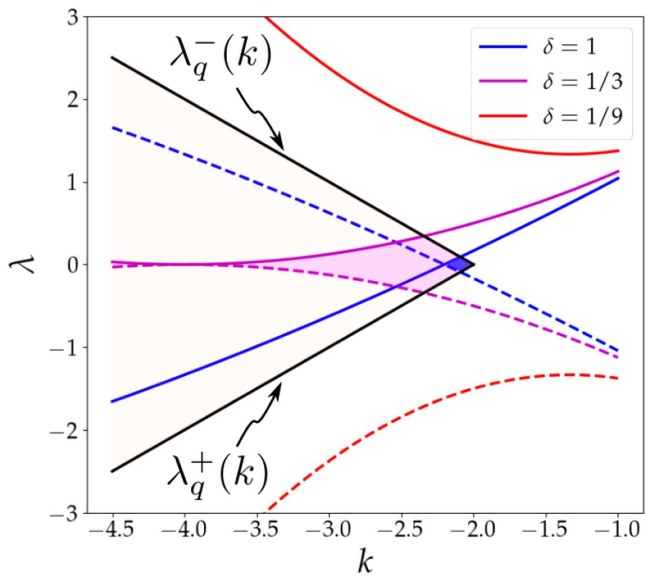

(b)

FIG. 10. (a) Two-parameter bifurcation diagram for the three-timescale Koper model [Eq. (1)] to leading order in $\varepsilon$ and $\delta$ : oscillatory dynamics is restricted to the triangular region of the $(k, \lambda)$-plane that is bounded by $\lambda_{q}^{\mp}(k)$. Mixed-mode dynamics is separated from relaxation oscillation by the curves $\lambda_{r}^{\mp}(k)$; to leading order, the mixed-mode regime is subdivided into regions of either single or double epochs of SAOs at $k=-4$. The curves $\lambda_{q}^{\mp}(k)$ and $\lambda_{r}^{\mp}(k)$ shown here are obtained in the singular limit of $\varepsilon=0=\delta$. Numerical verification of the cases corresponding to the colored shapes for $\varepsilon, \delta>0$ small is given in Fig. 11. (b) Classification of the folded singularities $q^{\mp}$ in dependence of $\delta$ : dashed and solid curves correspond to $q^{-}$and $q^{+}$, respectively, being of folded degenerate node type. For $\delta$ fixed, shading indicates parameter regimes in the $(k, \lambda)$-plane, where both $q^{\mp}$ are folded nodes. With decreasing $\delta$, these regimes stretch until the curves "detach" at $\delta=\frac{1}{3}$ (see Fig. 16 of Ref. 6 for comparison).

Sec. III, in particular, in relation to the classification in Fig. 6; the requisite calculations are simplified due to the symmetry of (1) by Remark 5.

In a first step, we note that the boundary between steady-state behavior and oscillatory dynamics in the Koper model is marked by curves that are $\mathcal{O}(\varepsilon, \delta)$-close to the lines given by $\lambda_{q}^{\mp}(k)=\mp(2+k)$; these are found by making use of (51) in (32) and solving for the $\lambda$-values for which a true equilibrium crosses either $q^{-}$or $q^{+}$, with $k$ fixed, corresponding to folded saddle-node bifurcations of type II. ${ }^{21,29}$ Since, in particular, the equilibria above the curve defined by $\lambda_{q}^{-}$and below the one defined by $\lambda_{q}^{+}$are stable, there can be no oscillatory dynamics for $k \in(-2,0)$, where at least one stable equilibrium will be present. It hence follows that oscillatory dynamics is restricted to the triangular area illustrated in Fig. 10 for $k<-2$. A further subdivision of that area is obtained by noting that mixed-mode dynamics is separated from relaxation oscillation by two curves $\lambda_{r}^{-}(k)$ and $\lambda_{r}^{+}(k)=-\lambda_{r}^{-}(k)$, which are found by substituting (51) into (41) and solving for $\lambda$ (we recall that, for the corresponding $\lambda$-values, the reduced flow on $\mathcal{S}^{a^{-}}$is balanced by that on $\mathcal{S}^{a^{+}}$at $q^{-}$and $q^{+}$, respectively; cf. also Theorem 2). While analytical expressions for $\lambda_{r}^{\mp}(k)$ can be obtained by direct integration, they are quite involved algebraically, and are hence not included here. These expressions imply that, for $\varepsilon=0=\delta$ and $k<-4, \lambda_{q}^{+}(k)$ $<\lambda_{r}^{+}(k)<\lambda_{r}^{-}(k)<\lambda_{q}^{-}(k)$, as well as that $\lambda_{r}^{\mp}$ are asymptotically parallel to $\lambda_{q}^{\mp}$, respectively, for sufficiently large $|k|$; moreover, the curves $\lambda_{r}^{\mp}(k)$ connect tangentially at $k=-4$ [numerically, one finds that, for $\varepsilon=\mathcal{O}\left(10^{-4}\right)$ and $\delta=\mathcal{O}\left(10^{-2}\right)$, the transition between mixed-mode dynamics and relaxation occurs at $\lambda_{r}^{\mp}(k)+\mathcal{O}(\delta)$, as is to be expected from (41)].

Finally, the resulting, chevron-shaped region in which mixedmode dynamics is observed is further divided into subregions in which either single or double epochs of SAOs are found. To leading order in $\varepsilon$ and $\delta$, that division occurs at $k=-4$. Geometrically, it is due to the fact that the folded singularities $q^{\mp}$ in the Koper model are remote for $k<-4$, while they are connected when $-4<k<0$ (recall Proposition 5 as well as Theorems 1 and 3, respectively). We emphasize that, in the two-timescale context of sufficiently small $\varepsilon$ and $\delta=\mathcal{O}(1)$, MMOs with double epochs of SAOs occur in a very narrow region of the $(k, \lambda)$-plane, as shown in Fig. 10(b) for $\delta=1$ (shaded blue). That region corresponds to the regime where both folded singularities $q^{\mp}$ are of folded node type and trajectories are attracted to both of them through the associated funnels, by Ref. 6; these funnels stretch as $\delta$ decreases (recall Lemma 1). Hence, in the three-timescale context, trajectories can reach both folded singularities $q^{\mp}$ as long as they are attracted to $\mathcal{M}_{2}$ on both $\mathcal{S}^{a^{\mp}}$, i.e., as long as $q^{-}$and $q^{+}$are aligned or connected.

Remark 7. Comparing Fig. 6 with Fig. 10, we note that the two panels in the former are combined in the latter, as one-parameter diagrams (in $\mu$ ) are merged into one two-parameter diagram in $(k, \lambda)$; correspondingly, parallel lines with $\mu$ constant in Fig. 6 are "bent," and hence intersect, in Fig. 10. (Here, we reiterate that $k$ determines the singular geometry of the Koper model, while $\lambda$ affects the resulting flow.) 


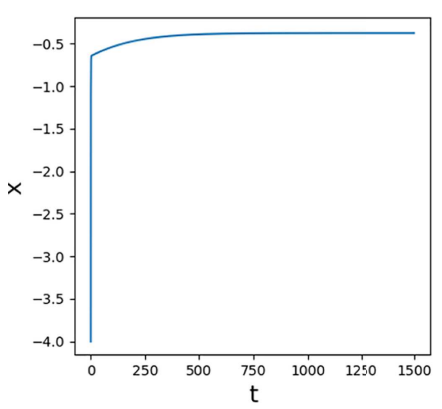

(a)

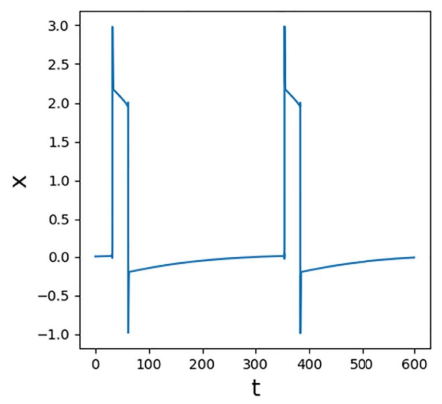

(c)

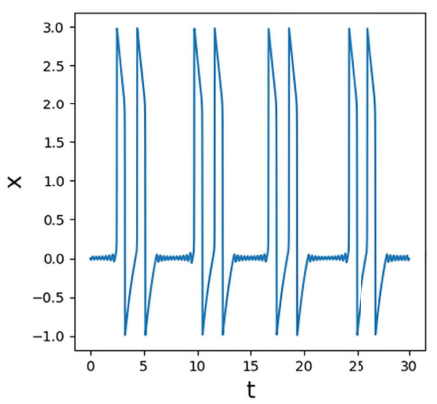

(e)

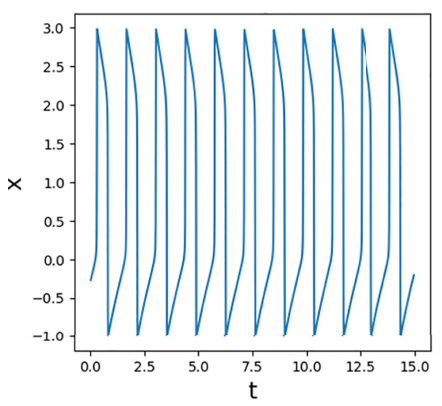

(g)

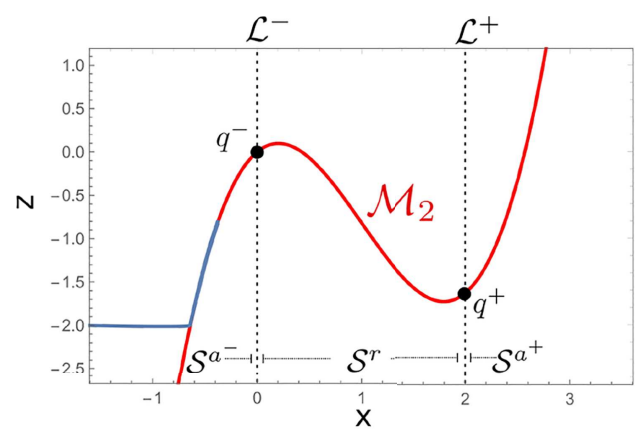

(b)

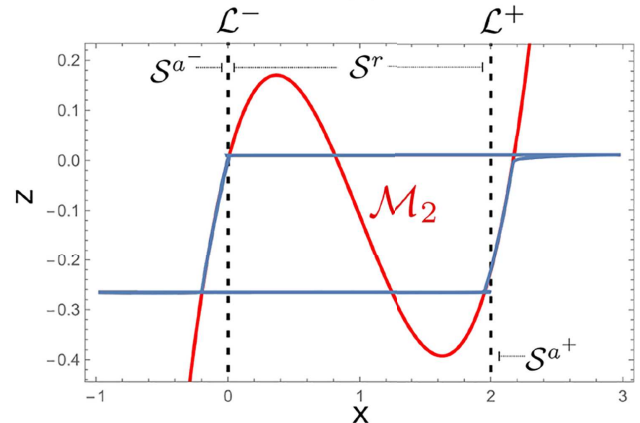

(d)

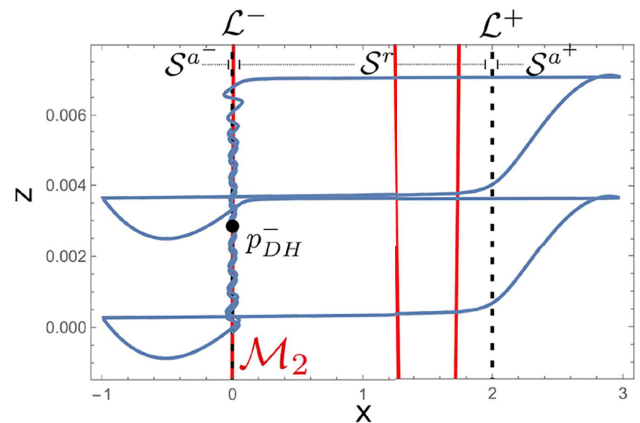

(f)

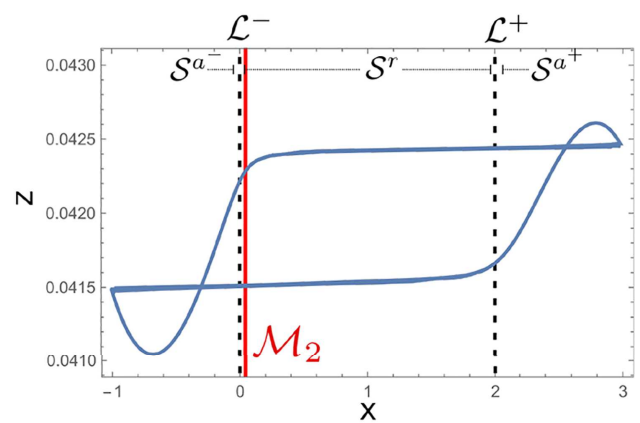

(h)

FIG. 11. Verification of the bifurcation diagram in Fig. 10 for representative choices of $k$, with $\varepsilon=0.01=\delta$ and $\lambda=1.5$ fixed: as $k$ decreases, one observes a transition from (a) steady-state behavior via (c) MMO trajectories with double epochs of SAOs and (e) single epochs of SAOs to (g) relaxation oscillation. The corresponding singular geometry in phase space is shown in panels $(b),(d),(f)$, and $(h)$. 


\section{Numerical verification}

In this subsection, we verify our classification of the threetimescale dynamics of the Koper model for various representative choices of the parameters $k$ and $\lambda$, as indicated in Fig. 10. We initially fix $\varepsilon=0.01=\delta$ and $\lambda=1.5$, and we vary $k$. We recall that the Koper model is symmetric in $\lambda$, and that it hence suffices to consider positive $\lambda$-values (cf. again Remark 5 and Fig. 1).

For $k=-2.2$ (red circle), the flow of the Koper model converges to steady state [see panel (a) of Fig. 11]. For $k=-3.6$ (green asterisk), we observe mixed-mode dynamics with double epochs of SAOs since the folded singularities $q^{\mp}$ are connected in that regime; the points at which these trajectories "jump" are estimated in Proposition 7 of the Appendix. We note that the dynamics on $\mathcal{Z}^{-}$differs from that on $\mathcal{Z}^{+}$due to the definition of $\phi(x, y, z)$ as given in (3), in spite of the singular geometry being symmetric [see Fig. 11(c)]. For $k=-4.4$ (blue diamond), the Koper model exhibits mixed-mode dynamics with single epochs of SAOs, as illustrated in panel (e) of Fig. 11. Finally, for $k=-5.4$ (purple triangle), we observe relaxation oscillation [see Fig. 11(c)].

It was shown in Ref. 4 that for $\delta=\mathcal{O}\left(\varepsilon^{2}\right)$, their prototypical model [Eq. (4)] can admit MMO trajectories which contain SAO segments that are the product of bifurcation delay alternating with sector-type dynamics. In Fig. 12, we present an example that indicates sector-delayed-Hopf-type dynamics in the Koper model; as indicated in Sec. III C, a crude requirement for the existence of such mixed dynamics is that $\delta=\mathcal{O}\left(\varepsilon^{c}\right)$ for $c \geq 1$. We remark that sectortype SAOs cease to exist when $k=-4$, as the corresponding regions on $\mathcal{Z}^{\mp}$ vanish then (see Lemma 4 in the Appendix for details).

We emphasize again that the MMO trajectories described here cannot be viewed, strictly speaking, as perturbations of individual singular cycles, as defined in Sec. II. Rather, we have shown that if the folded singularities of (2) are remote, then there exist $\varepsilon$ and $\delta$ positive and sufficiently small such that the Koper model exhibits MMOs with single epochs of SAOs; correspondingly, we

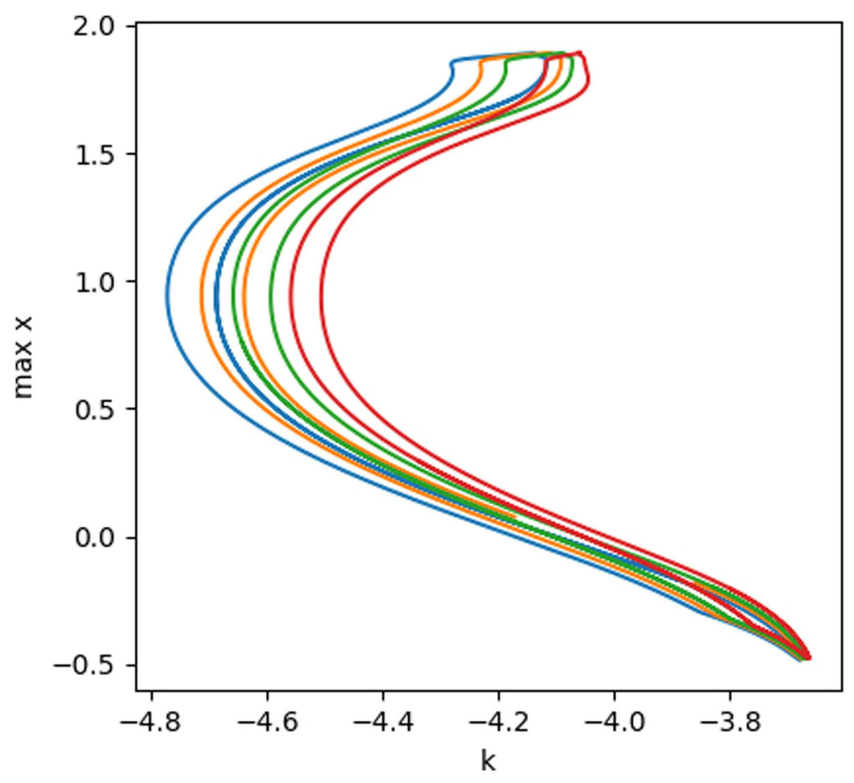

FIG. 13. Numerical continuation of periodic orbits in the Koper model with auto-07p $p^{8}$ for $\lambda=1.5$ and $\varepsilon=0.1=\delta$ : one observes the coexistence of multiple periodic orbits, as evidenced by the overlap between the corresponding $k$-intervals; the overlapping plateaus correspond to stable periodic orbits.

observe double epochs of SAOs if those singularities are aligned or connected. The above statement is corroborated by numerical continuation, as illustrated in Fig. 13, where multiple periodic orbits seem to coexist for $k, \lambda, \varepsilon$, and $\delta$ fixed; we emphasize that this continuation is quite delicate, even for the relatively large values of $\varepsilon$ and $\delta$ chosen here [a similar observation was made in the context of

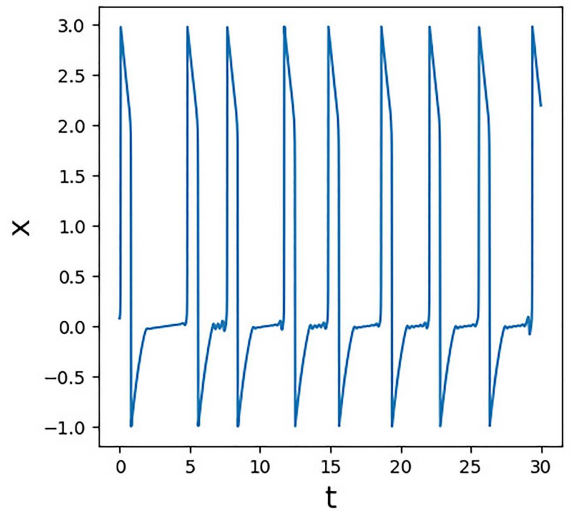

(a)

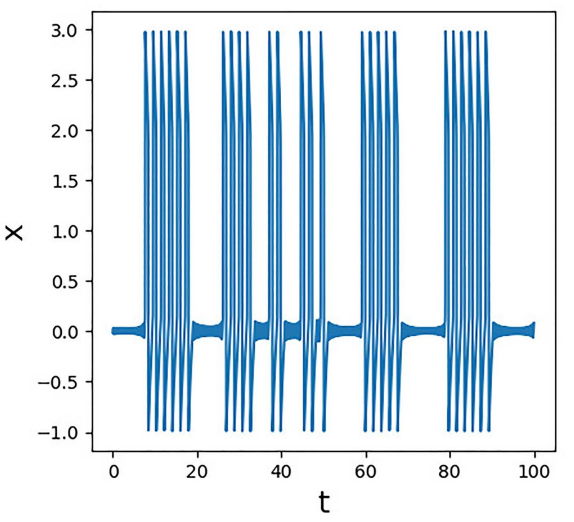

(b)

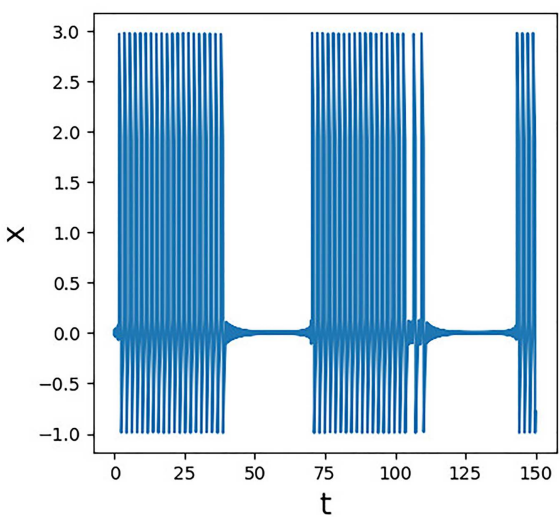

(c)

FIG. 12. Mixed-mode time series in the Koper model for $\varepsilon=0.01$ fixed and varying $\delta$ : as $\delta$ decreases, the number of LAOs between SAO segments typically increases; additionally, for these specific parameter values, the model seems to exhibit sector-delayed-Hopf-type dynamics, ${ }^{4}$ as is particularly apparent in panel (b). (a) $\delta=0.1$ $=\mathcal{O}(\sqrt{\varepsilon})$. (b) $\delta=0.001=\mathcal{O}\left(\varepsilon^{\frac{3}{2}}\right)$. (c) $\delta=0.0003=\mathcal{O}\left(\varepsilon^{2}\right)$. 
the two-timescale Koper model, i.e., for $\delta=1$ in Eq. (1c) (Fig. 19 of Ref. 6)]. An in-depth study of the properties of these periodic orbits in relation to the mixed-mode dynamics of Eq. (2) is left for future work.

\section{CONCLUSIONS}

In the present article, we have introduced an extended prototypical example of a three-dimensional, three-timescale system [Eq. (2)]. We have classified the mixed-mode dynamics of that system in dependence of its parameters, thus relating bifurcations of MMO trajectories to the underlying singular geometry. In particular, in Sec. III, we identified the geometric mechanism that is responsible for the transition from MMOs with single epochs of SAOs to those with double epochs, and we argued that the latter are robust in the three-timescale context. Specifically, we showed that, if the folded singularities of (2) are remote, then there exist sufficiently small $\varepsilon$ and $\delta$ such that our system exhibits either MMOs with single epochs of SAOs or two-timescale relaxation oscillation, whereas double epochs of SAOs can be observed if the singularities are aligned or connected (cf. Proposition 3). In Sec. IV, we demonstrated our results for the Koper model from chemical kinetics, ${ }^{17}$ which represents one particular realization of (2); in particular, we constructed the two-parameter bifurcation diagram in Fig. 10 on the basis of results obtained in Sec. III, thus classifying in detail the mixed-mode dynamics of the three-timescale Koper model.

A posteriori, it is evident that the local dynamics of our extended prototypical model [Eq. (2)] is similar to that of the canonical system [Eq. (5)] proposed in Ref. 23; however, due to the absence of a cubic $x$-term in (5a), the latter can yield SAO-type dynamics only due to the lack of an LAO-generating global return mechanism. The prototypical system in Eq. (4), on the other hand, does not allow for the 2-critical manifold to be cubic-like due to the $y$-term being absent in (4b) and can hence only exhibit MMOs with single epochs of SAOs, as opposed to our Eq. (2) (recall Proposition 3). Hence, we postulate that the extended prototypical model in (2) represents the simplest general example within that given class of systems that can encapsulate the geometric mechanism described in Sec. III. Here, we emphasize that we have not considered explicitly the scenario where the fold points $p^{\mp}$ of $\mathcal{M}_{2}$ can cross the fold lines $\mathcal{L}^{\mp}$ of $\mathcal{M}_{1}$ [recall, in particular, panels (b) and (e) of Fig. 4]. While that scenario is not realized in the Koper model [Eq. (1)], it has been shown to give rise to interesting global dynamics through the interaction of $p^{\mp}$ with $\mathcal{L}^{\mp}$; a recent, relevant example can be found in Ref. 7 . Additionally, we remark that the singular geometry considered here is relatively specific due to its symmetry properties; a generalization to asymmetric geometries may be of interest. The case illustrated in Fig. 4(a), for instance, is expected to feature relaxation oscillation rather than mixed-mode dynamics; if, however, the symmetry were broken, with $p^{-}$situated in $\mathcal{S}^{r}$ and $p^{+}$in $\mathcal{S}^{a^{+}}$, then one may expect to observe MMO trajectories with SAOs below but no SAOs above.

Our analysis in Sec. III shows that, in parameter regimes where both $\mathcal{M}_{1}$ and $\mathcal{M}_{2}$ are normally hyperbolic, standard GSPT ${ }^{10}$ implies that an iterative reduction of timescales can be applied. In the fully perturbed Eq. (2), with $\varepsilon$ and $\delta$ being sufficiently small, it follows that the manifolds $\mathcal{Z}_{\varepsilon \delta}^{\mp, r}$ lie $\mathcal{O}(\delta)$-close to their unperturbed counterparts $\mathcal{Z}^{\mp, r}$, respectively, since $\mathcal{Z}_{\varepsilon 0}^{\mp, r}$ are $\varepsilon$-independent. Since, moreover, the manifolds $\mathcal{S}_{\varepsilon 0}^{a, r}$ lie $\mathcal{O}(\varepsilon)$-close to $\mathcal{S}^{a, r}$, ${ }^{10}$ any fibers of $\mathcal{Z}_{\varepsilon \delta}^{\mp, r}$ that lie on $\mathcal{S}_{\varepsilon \delta}^{a, r}$ are $\mathcal{O}(\varepsilon+\delta)$-close to $\mathcal{S}^{a, r}$ (that estimate is in disagreement with Ref. 1; however, we note that, away from $\mathcal{Z}_{\varepsilon \delta}^{\mp, r}, \mathcal{S}_{\varepsilon \delta}^{a, r}$ are $\mathcal{O}(\varepsilon)$ close to $\mathcal{S}^{a, r}$ ). Under Assumption 2, trajectories that are attracted to $\mathcal{Z}_{\varepsilon \delta}^{\mp, r}$ follow the slow flow of (17) and potentially undergo SAOs. In the context of (2), the mechanisms that generate these SAOs are "bifurcation delay" Appendix for details).

With regard to regions where normal hyperbolicity of $\mathcal{M}_{1}$ is lost, we reiterate that the dynamics of Eq. (2) combines features of two-timescale slow-fast systems with either two slow variables and a fast one, or one fast variable and two slow ones. As shown in Sec. III, the corresponding mechanisms hence coexist and interact, giving rise to complex local dynamics in the vicinity of the fold lines $\mathcal{L}^{\mp}$ in (2). We briefly sketched the implications of that interaction; in particular, in the Appendix, we relate the emergence of canard-type SAOs to the perturbation of an integrable system. ${ }^{18} \mathrm{~A}$ more rigorous description of the resulting near-integrable system

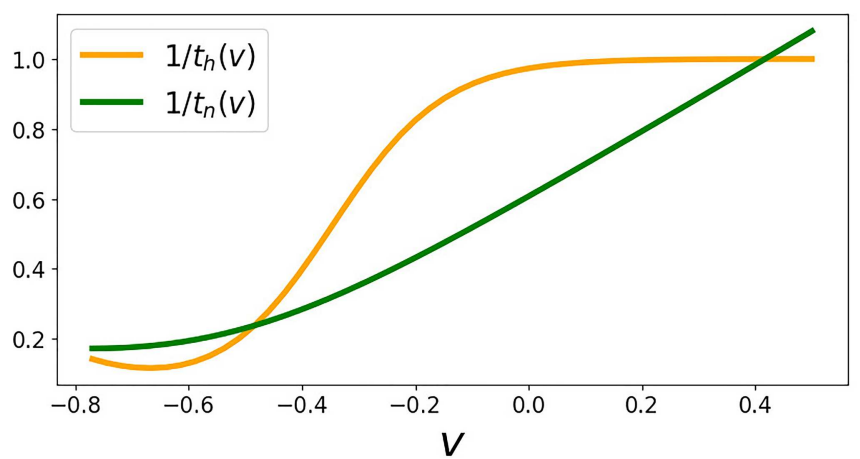

(a)

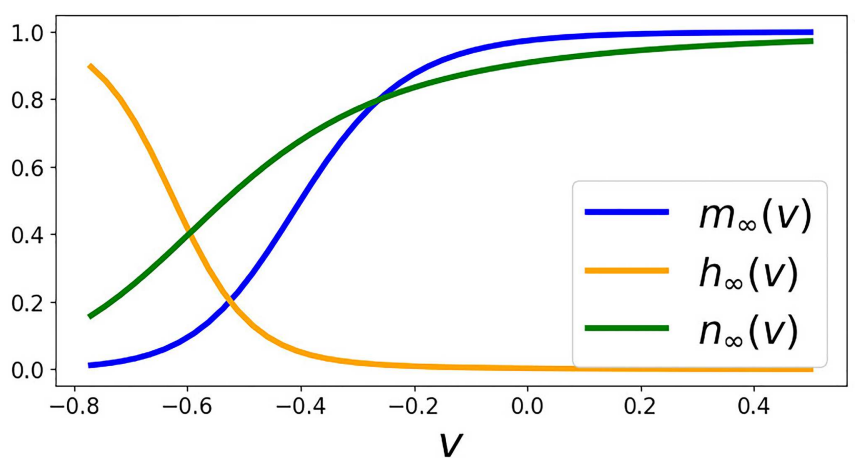

(b)

FIG. 14. Representative graphs of the nonlinear functions on the right-hand sides of the Hodgkin-Huxley equations in (56). (a) $\frac{1}{t_{h}(v)}$ and $\frac{1}{t_{n}(v)}$. (b) $x_{\infty}(v)(x=m, h, n)$. 


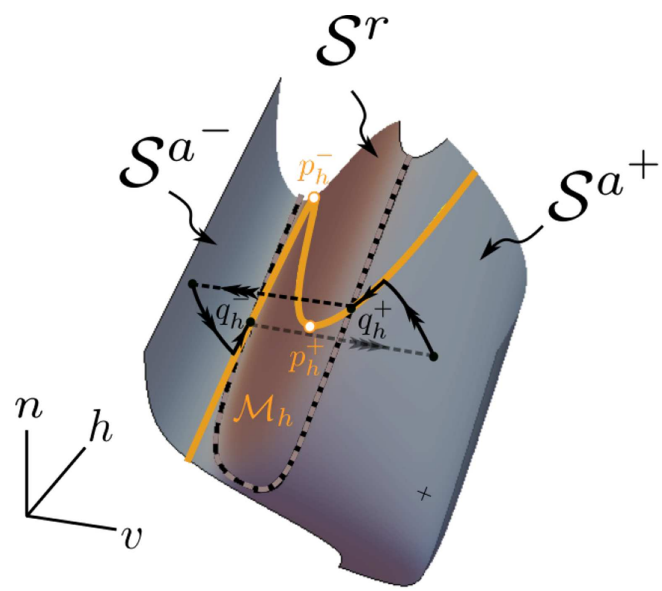

(a)

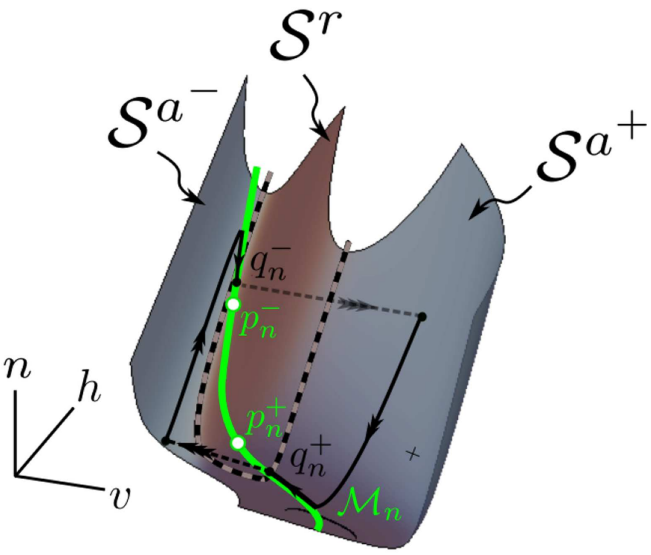

(b)

FIG. 15. The critical and 2-critical manifolds of the three-dimensional, three-timescale Hodgkin-Huxley model [Eq. (56)] when either $h$ or $n$ is taken to be the slowest variable [see panels (a) and (b), respectively]. Singular cycles that pass through both folded singularities are shown; in both cases, the corresponding perturbed dynamics will feature MMO trajectories with double epochs of SAOs. A more detailed analysis of the multi-timescale Hodgkin-Huxley equations within the framework of GSPT is given in an upcoming work. ${ }^{16}$ (a) $\tau_{h} \gg 1$ and $\tau_{n}=1$. (b) $\tau_{h}=1$ and $\tau_{n} \gg 1$.

in the context of the Koper model [Eq. (1)], is part of work in progress. Of particular interest here is the investigation of Shilnikovtype homoclinic phenomena, ${ }^{12,25}$ as well as the further classification of MMOs with single epochs of SAOs; specifically, we conjecture that the bifurcation diagram in Fig. 10 may be refined in that one can identify regions of chaotic mixed-mode dynamics in dependence of the various parameters in the model, as well as of the ratio of $\varepsilon$ and $\delta$.

We emphasize that, strictly speaking, the MMO trajectories described in Sec. III cannot be considered as perturbations, for positive $\varepsilon$ and $\delta$, of the individual singular cycles constructed in Sec. II. Rather, the latter determine the qualitative properties of the former, for sufficiently small $\varepsilon$ and $\delta$, as is evident from Fig. 13 in the context of the three-scale Koper model, where several periodic orbits seem to coexist for a given choice of $k, \lambda, \varepsilon$, and $\delta$.

Finally, we emphasize that the geometric mechanism described in this article extends beyond the Koper model from chemical kinetics studied in Sec. IV. One prominent example of a rich multiplescale system that features similar geometric properties as our prototypical model [Eq. (2)] is provided by a three-dimensional reduction of the famous Hodgkin-Huxley equations from mathematical neuroscience, ${ }^{28}$

$$
\epsilon \dot{v}=\bar{I}-\left(v-\bar{E}_{N a}\right) m_{\infty}(v)^{3} h-\bar{g}_{k}\left(v-\bar{E}_{k}\right) n^{4}-\bar{g}_{l}\left(v-\bar{E}_{L}\right),
$$

$$
\begin{aligned}
& \dot{h}=\frac{1}{\tau_{h} t_{h}(v)}\left(h_{\infty}(v)-h\right), \\
& \dot{n}=\frac{1}{\tau_{n} t_{n}(v)}\left(n_{\infty}(v)-n\right),
\end{aligned}
$$

where $v$ is the fast variable, while $h$ and $n$ are the slow ones. Here, the functions $\frac{1}{t_{h}(v)}, \frac{1}{t_{n}(v)}$, and $x_{\infty}(v)(x=m, h, n)$ illustrated in Fig. 14 are defined as in Ref. 28, as are the values of the various parameters in Eq. (56) (see also Ref. 9). In particular, following Ref. 9, we may set $\tau_{n}=1$ in (56) and assume that $\tau_{h} \gg 1$ is sufficiently large; alternatively, we may take $\tau_{h}=1$ and $\tau_{n} \gg 1$. In either case, we obtain a three-timescale system, where $v$ is the fast variable, with $n$ and $h$ being intermediate or slow, respectively.

Figure 15 indicates that the resulting singular geometry of Eq. (56) is analogous to that of our extended prototypical example [Eq. (2)] (recall Fig. 5). MMO trajectories can, hence, again be constructed as outlined in Sec. III, by combining segments that evolve on separate timescales. Upon variation of the parameter $\bar{I}$-the (rescaled) applied current in the Hodgkin-Huxley formalism-transitions between MMOs with different qualitative properties occur via a mechanism that is similar to the one described for Eq. (2) in Secs. II and III. For an in-depth geometric analysis of a novel, global three-dimensional reduction of the multi-timescale Hodgkin-Huxley equations, rather than of Eq. (56), the reader is referred to the upcoming work, Ref. 16.

\section{ACKNOWLEDGMENTS}

The authors thank Martin Krupa and Martin Wechselberger for their critical reading of previous versions of the manuscript and for constructive feedback as well as for insightful discussions and relevant references. The authors are also grateful to two anonymous reviewers whose comments and suggestions greatly improved the original manuscript. P.K. was supported by the Principal's Career Development Scholarship for Ph.D. studies of the University of Edinburgh. 


\section{AUTHOR DECLARATIONS}

\section{Conflict of Interest}

The authors have no conflicts of interest to disclose.

\section{DATA AVAILABILITY}

Data sharing is not applicable to this article as no new data were created or analyzed in this study.

\section{APPENDIX: SAO-GENERATING MECHANISMS}

In this appendix, we briefly discuss the local, SAO-type dynamics of our prototypical model [Eq. (2)]; specifically, we give an overview of two SAO-generating mechanisms-bifurcation delay and sector-type dynamics-within the framework of (2).

\section{Local dynamics and SAOs}

We begin by discussing the emergence of SAOs in a vicinity of $\mathcal{L}^{\mp}$ in (2) when trajectories are attracted to $\mathcal{Z}^{\mp}$, respectively; we focus on describing the properties of $\mathcal{Z}^{-}$close to $\mathcal{L}^{-}$here, as the description of $\mathcal{Z}^{+}$near $\mathcal{L}^{+}$is analogous.

We first consider the partially perturbed fast Eq. (6) with $\varepsilon$ being sufficiently small and $\delta=0$,

$$
\begin{gathered}
x^{\prime}=-y+f_{2} x^{2}+f_{3} x^{3}, \\
y^{\prime}=\varepsilon(\alpha x+\beta y-z), \\
z^{\prime}=0 .
\end{gathered}
$$

By standard GSPT, ${ }^{10,19}$ we can define slow manifolds $\mathcal{S}_{\varepsilon 0}^{a, r}$ for (A1) as surfaces that are foliated by orbits within $\left\{z=z_{0}\right\}$, with $z_{0}$ constant. Since the steady states of (A1) correspond to portions of the 2-critical manifold $\mathcal{M}_{2}$, it follows that $\mathcal{Z}_{\varepsilon 0}^{\mp, r} \equiv \mathcal{Z}^{\mp, r}$, i.e., that the geometry of $\mathcal{Z}_{\varepsilon 0}^{\mp, r}$ is, in fact, $\varepsilon$-independent. However, since it will become apparent that the stability properties of $\mathcal{Z}_{\varepsilon 0}^{\mp, r}$ do depend on $\varepsilon$, we will not suppress the $\varepsilon$-subscript in our notation.

For sufficiently small $\varepsilon$, Eq. (A1) undergoes a Hopf bifurcation at a point $p_{D H}^{-}=\left(x_{D H}^{-}, y_{D H}^{-}, z_{D H}^{-}\right)$; the periodic orbits that arise in that bifurcation cease to exist at $z_{C N}^{-}$, where a connecting trajectory between the manifolds $\mathcal{S}_{\varepsilon 0}^{a^{-}}$and $\mathcal{S}_{\varepsilon 0}^{r}$ is found. In other words, $\mathcal{S}_{\varepsilon 0}^{a^{-}}$and $\mathcal{S}_{\varepsilon 0}^{r}$ intersect transversely within the hyperplane $\mathcal{P}_{C N}^{-}$:
$\left\{z=z_{C N}^{-}\right\}$, which lies $\mathcal{O}(\varepsilon)$-close to $p_{D H}^{-}$in the $z$-direction. Moreover, two degenerate nodes $p_{D N_{\mp}}^{-}$are located on $\mathcal{M}_{2}$ around $p_{D H}^{-}$ at an $\mathcal{O}(\sqrt{\varepsilon})$-distance (see Fig. 16). The asymptotics (in $\varepsilon$ ) of these objects is summarized below.

Lemma 4. A Hopf bifurcation of Eq. (A1) occurs at $p_{D H}^{-}$ $=\left(x_{D H}^{-}, y_{D H}^{-}, z_{D H}^{-}\right) \in \mathcal{Z}_{\varepsilon 0}^{-}$, where

$$
\begin{gathered}
x_{D H}^{-}=-\frac{\beta}{2 f_{2}} \varepsilon+\mathcal{O}\left(\varepsilon^{2}\right), \quad y_{D H}^{-}=\frac{\beta^{2}}{4 f_{2}} \varepsilon^{2}+\mathcal{O}\left(\varepsilon^{3}\right), \quad \text { and } \\
z_{D H}^{-}=-\frac{\alpha \beta}{2 f_{2}} \varepsilon+\mathcal{O}\left(\varepsilon^{2}\right) .
\end{gathered}
$$

Two degenerate nodes $p_{D N_{\mp}}^{-}$are located at

$$
\begin{gathered}
x_{D N_{\mp}}^{-}=\mp\left(\frac{\sqrt{\alpha}}{f_{2}} \sqrt{\varepsilon}+\frac{\beta}{2 f_{2}} \varepsilon\right)+\mathcal{O}\left(\varepsilon^{\frac{3}{2}}\right), \\
y_{D N_{\mp}}^{-}=\frac{\alpha}{f_{2}} \varepsilon+\mathcal{O}\left(\varepsilon^{\frac{3}{2}}\right), \quad \text { and } \\
z_{D N_{\mp}}^{-}=\mp \frac{\alpha^{\frac{3}{2}}}{f_{2}^{2}} \sqrt{\varepsilon}+\frac{\alpha \beta}{f_{2}}\left(1 \mp \frac{1}{2}\right) \varepsilon+\mathcal{O}\left(\varepsilon^{\frac{3}{2}}\right),
\end{gathered}
$$

while a canard trajectory is contained in the hyperplane $\mathcal{P}_{C N}^{-}$: $\left\{z=z_{C N}^{-}\right\}$, with

$$
z_{C N}^{-}=z_{D H}^{-}+\alpha \beta \frac{5 f_{2}-3\left(1-\alpha f_{3}\right)}{4\left(1+f_{2}\right) f_{2}} \varepsilon+\mathcal{O}\left(\varepsilon^{2}\right) .
$$

Proof. The hyperplane $\left\{z=z_{C N}^{-}\right\}$, which contains the transverse intersection between $\mathcal{S}_{\varepsilon 0}^{a^{-}}$and $\mathcal{S}_{\varepsilon 0}^{r}$, can be obtained by Melnikov-type calculations (see Refs. 19 and 23). The remaining estimates follow by considering the Jacobian matrix of the linearization of (A1) along $\mathcal{M}_{2}$,

$$
J=\left(\begin{array}{cc}
x\left(2 f_{2}+3 f_{3} x\right) & -1 \\
\varepsilon \alpha & \varepsilon \beta
\end{array}\right),
$$

the eigenvalues of which are

$$
\begin{aligned}
v_{1,2}= & \frac{1}{2}\left[\beta \varepsilon+2 f_{2} x+3 f_{3} x^{2}\right. \\
& \left. \pm \sqrt{\left(\beta \varepsilon+2 f_{2} x+3 f_{3} x^{2}\right)^{2}-4\left(\alpha \varepsilon+2 \beta \varepsilon f_{2} x+3 \beta \varepsilon f_{3} x^{2}\right)}\right] .
\end{aligned}
$$

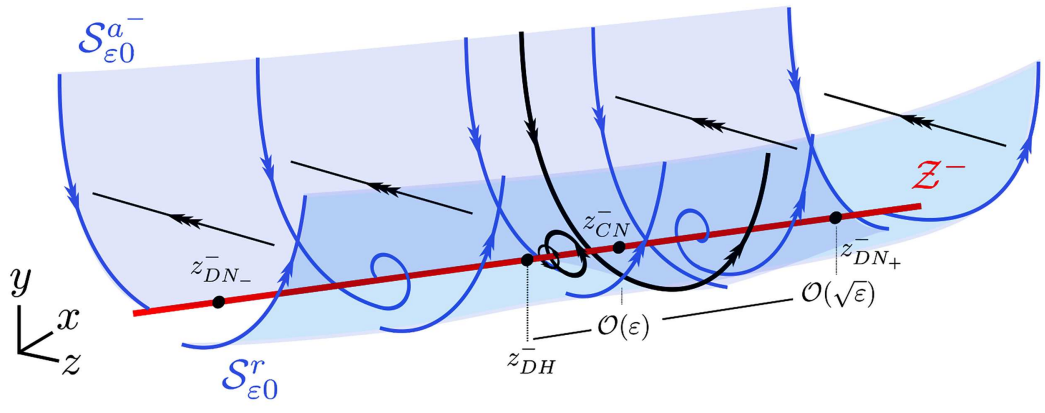

FIG. 16. Stability of the 2-critical manifold $\mathcal{M}_{2}$ on the various portions of $\mathcal{Z}^{-}$for sufficiently small $\varepsilon$ and $\delta=0$. At $z_{D N_{-}}^{-}$, the real eigenvalues of the linearization of Eq. (A1) about $\mathcal{M}_{2}$ in (A6) become complex, with a corresponding change from nodal to focal attraction or repulsion, and vice versa; at $z_{D H}^{-}$, a (supercritical) Hopf bifurcation occurs which gives rise to small-amplitude periodic orbits. These orbits cease to exist at $\mathrm{Z}_{\mathrm{CN}}^{-}$, where a connecting trajectory between $\mathcal{S}_{\varepsilon 0}^{a^{-}}$and $\mathcal{S}_{\varepsilon 0}^{r}$ is found. We note that the corresponding $z$-interval is of width $\mathcal{O}(\varepsilon)$, while the focal region is $\mathcal{O}(\sqrt{\varepsilon})$ wide overall (the geometry due to a subcritical Hopf bifurcation can be visualized in a similar fashion). 
Remark 8. The Hopf bifurcation at $p_{D H}^{-}$is "inherited" from the fact that $\mathcal{M}_{2}$ and $\mathcal{L}^{-}$intersect in the folded singularity $q^{-}$: for $\varepsilon=0=\delta$, the trace of the Jacobian matrix J vanishes at that point.

Remark 9. The estimate in (A4) is a generalization of the corresponding expression in Ref. 23 for their canonical system [Eq. (5)]; the $f_{3}$-dependence of (A4) implies that the cubic $x$-terms in our Eq. (2a) do, in fact, contribute to the local dynamics.

Motivated by Lemma 4, we introduce the following notation: for $\delta=0$, we define the intervals

$$
\begin{gathered}
\mathcal{I}_{\text {nod }}=\left(-\infty, z_{D N_{-}}^{-}\right), \quad \mathcal{I}_{\text {foc }}=\left(z_{D N_{-}}^{-}, z_{D H}^{-}\right), \quad \text { and } \\
\mathcal{I}_{\text {can }}=\left(\min \left\{z_{D H}^{-}, z_{C N}^{-}\right\}, \max \left\{z_{D H}^{-}, z_{C N}^{-}\right\}\right) .
\end{gathered}
$$

Then, it follows that

1. the manifold $\mathcal{S}_{\varepsilon 0}^{a^{-}}$connects to $\mathcal{Z}^{-}$for $z<\min \left\{z_{D H}^{-}, z_{C N}^{-}\right\}$, while $\mathcal{S}_{\varepsilon 0}^{r}$ connects to $\mathcal{Z}^{-}$for $z>\max \left\{z_{D H}^{-}, z_{C N}^{-}\right\}$;

2. for $f_{2}<\frac{3}{5}\left(1-\alpha f_{3}\right)$, i.e., for $z_{C N}^{-}>z_{D H}^{-}$, the Hopf bifurcation at $p_{D H}^{-}$is supercritical, with the resulting periodic orbits the $\omega$-limit sets of trajectories on $\mathcal{S}_{\varepsilon 0}^{a^{-}}$; and

3. for $f_{2}>\frac{3}{5}\left(1-\alpha f_{3}\right)$, i.e., for $z_{C N}^{-}<z_{D H}^{-}$, the Hopf bifurcation at $p_{D H}^{-}$is subcritical, with the resulting periodic orbits the $\alpha$-limit sets of trajectories on $\mathcal{S}_{\varepsilon 0}^{r}$.

The corresponding geometry is illustrated in Fig. 16; we emphasize that analogous objects $p_{D H}^{+}, \mathcal{P}_{C N}^{+}$, and $p_{D N_{ \pm}}^{+}$, which are located symmetrically to the above, exist on $\mathcal{Z}^{+}$.

We define the canard point $p_{C N}^{-}=\left(x_{C N}^{-}, y_{C N}^{-}, z_{C N}^{-}\right)$by

$$
p_{C N}^{-}=\mathcal{P}_{C N}^{-} \cap \mathcal{Z}^{-}
$$

It has already been pointed out in Ref. 23 that $p_{C N}^{-}$and the Hopf point $p_{D H}^{-}$on $\mathcal{Z}^{-}$collapse to the origin in the limit of $\varepsilon=0$; correspondingly, the origin is referred to as the "canard delayed Hopf singularity" in the double singular limit of $\varepsilon=0=\delta$. As a result, the folded singularity at $q^{-}$displays characteristics of both a Hopf point-in that the trace of the Jacobian in (A5) vanishes-and a canard point-in that $\mathcal{S}^{a^{-}}$and $\mathcal{S}^{r}$ meet along a fold. Moreover, we remark that an "incomplete" canard explosion ${ }^{5}$ occurs at $z_{C N}^{-}$in Eq. (5), as the corresponding intermediate problem has two equilibria, with the equilibrium corresponding to $\mathcal{Z}^{r}$ being a saddle forming a homoclinic connection to itself (see Ref. 23 for details). On the other hand, Eq. (2) could feature either a complete or an incomplete canard explosion, depending on the relative position of $q^{-}$and $p^{+}$; the implications thereof for the global dynamics are currently being investigated.

We briefly describe the associated two mechanismsbifurcation delay and sector-type dynamics-in the following; we remark that the former is common in two-timescale systems with two fast variables, while the latter typically occurs in two-timescale systems with two slow variables. Therefore, the coexistence of these mechanisms in three-timescale systems is due to the fact that such systems can simultaneously be viewed as having two fast and one slow variables, as well as one fast and two slow variables (for four- dimensional two-timescale systems with two fast and two slow variables that interplay has been documented in Ref. 2).

\section{Bifurcation delay}

Bifurcation delay is typically encountered in two-timescale systems with two fast variables and one slow variable. In the context of Eq. (2), it is realized when trajectories are attracted to $\left.\mathcal{Z}_{\varepsilon \delta}\right|_{\mathcal{I}_{\text {nod }}+\mathcal{O}(\delta)}$ or $\left.\mathcal{Z}_{\varepsilon \delta}\right|_{\mathcal{I}_{\text {foc }}+\mathcal{O}(\delta)}$ [recall (A7) and Fig. 16]. Following the slow flow on $\mathcal{Z}_{\varepsilon \delta}^{-}$, trajectories experience a delay in being repelled from $\mathcal{Z}_{\varepsilon \delta}^{-}$ when crossing the Hopf bifurcation point $p_{D H}^{-}$, as the accumulated contraction to $\mathcal{Z}_{\varepsilon \delta}^{-}$needs to be balanced by the total expansion from $\mathcal{Z}_{\varepsilon \delta}^{-}{ }^{21}$ Specifically, given some point $p_{\text {in }}=\left(x_{\text {in }}, y_{\text {in }}, z_{\text {in }}\right)$ in an $\mathcal{O}(\delta)$-neighborhood of $\mathcal{Z}_{\varepsilon \delta}^{-}$, one obtains the $x$-coordinate $x_{\text {out }}$ of the corresponding point $p_{\text {out }}$ where the given trajectory through $p_{\text {in }}$ exits an $\mathcal{O}(\delta)$-neighborhood of $\mathcal{Z}_{\varepsilon \delta}^{+}$from

$$
\int_{x_{\text {in }}}^{x_{\text {out }}} \frac{\Re\left\{v_{1,2}(x)\right\}}{\mu+\phi(x, F(x), G(x))} \mathrm{d} x=0 .
$$

Here, $v_{1,2}$ are the eigenvalues of the linearization of Eq. (A1) about $\mathcal{Z}_{\varepsilon \delta}^{-}$, as defined in (A6). Trajectories that are attracted to $\left.\mathcal{Z}_{\varepsilon \delta}\right|_{\mathcal{I}_{\text {foc }}}$ typically exhibit "dense" SAOs with initially decreasing and then increasing amplitude; see panel (e) of Fig. 11 for an illustration in the context of the Koper model [Eq. (1)]. By contrast, trajectories that are attracted to $\left.\mathcal{Z}_{\varepsilon \delta}\right|_{\mathcal{I}_{\text {nod }}}$ are characterized by very few SAOs that are followed by a large excursion [cf. Fig. 11(c)].

The case where trajectories enter the focally attracting region $\mathcal{I}_{\text {foc }}$ is naturally studied in the "rescaling chart" $\kappa_{2}$ which is introduced as part of a blow-up analysis in Refs. 19 and 23 since that region is bounded by the degenerate nodes $p_{D N_{\mp}}^{-}$and, thus, of width $\mathcal{O}(\sqrt{\varepsilon})$. In that case, the eigenvalues $v_{1,2}$ in (A6) are complex conjugates, which implies that the corresponding trajectory of (2) undergoes damped oscillation toward $\mathcal{Z}_{\varepsilon \delta}^{-}$.

On the other hand, when trajectories enter the nodally attracting region $\mathcal{I}_{\text {nod }}$, the corresponding entry point is typically $\mathcal{O}\left(\varepsilon^{c}\right)$ away from the folded singularity $q^{-}$, with $c<\frac{1}{2}$. One may, therefore, refer to the unscaled system [Eq. (2)] for the study of that case. The eigenvalues $v_{1,2}$ in (A6) correspond to strong and weak eigendirections: specifically, for $z<z_{D N_{-}}^{-}$, the eigenvalue $v_{1}$ represents the weak eigendirection, while the eigenvalue $v_{2}$ corresponds to the strong eigendirection; that correspondence is reversed for $z>z_{D N_{-}}^{+}$. Due to the hierarchy of timescales in (2), trajectories are first attracted to $\mathcal{S}_{\varepsilon \delta}^{a^{-}}$and then to $\mathcal{Z}_{\varepsilon \delta}^{-}$. Therefore, for initial conditions in $\mathcal{S}_{\varepsilon \delta}^{a^{-}}$, trajectories approach $\mathcal{Z}_{\varepsilon \delta}^{-}$along the weak eigendirection, whereas in $\mathcal{S}_{\varepsilon \delta}^{r}$, trajectories are repelled from $\mathcal{Z}_{\varepsilon \delta}^{-}$along the strong eigendirection. It is hence reasonable to balance the accumulated contraction and expansion using solely $v_{1}$ in (A8). Since the accumulated contraction on the intermediate timescale has to be balanced by expansion on the fast timescale, we have the following:

Proposition 7 (Refs. 13, 21, 26, and 27). Assume that Assumption 1 and Assumption 2 hold, and consider $p_{\text {in }}$

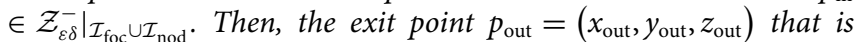


defined by (A8) satisfies

$$
\begin{gathered}
x_{\text {out }}<x_{D N_{+}}^{-}+o(1), \quad y_{\text {out }}<y_{D N_{+}}^{-}+o(1), \quad \text { and } \\
z_{\text {out }}<z_{D N_{+}}^{-}+o(1) .
\end{gathered}
$$

The above result is well-known; see, for instance, Corollary 2.3 of Ref. 13 for a brief discussion of its implications.

Remark 10. The estimates on the entry point $p_{\text {out }}$ in Proposition 7 can be refined under the additional assumption that the slow flow of Eq. (2) is constant, i.e., that $\phi(x, y, z)=0$ : as in Ref. 4 for $z_{\text {in }} \in \mathcal{I}_{\mathrm{foc}}^{\text {in }}$, it then follows from (A8) that $x_{\mathrm{out}}=x_{D H}-x_{\mathrm{in}}$.

Remark 11. In Ref. 23 for constant slow flow in Eq. (2), i.e., for $\phi(x, y, z)=0$, the weak contraction toward $\mathcal{Z}_{\varepsilon \delta}^{-}$is balanced by the weak expansion therefrom via

$$
\int_{x_{\text {in }}}^{x_{D N}^{-}} \Re\left\{v_{1}\right\} \mathrm{d} x+\int_{x_{D N}^{+}}^{x_{\text {out }}} \Re\left\{v_{2}\right\} \mathrm{d} x=0 .
$$

In that context, the fold point $p^{-}$was in fact identified as the buffer point at which trajectories have to leave $\mathcal{Z}_{\varepsilon \delta}^{-}$, which allows them to account for maximal canard trajectories.

\section{Sector-type dynamics}

Sector-type dynamics is typically encountered in two-timescale systems with one fast variable and two slow variables; it can be described by exploiting the near-integrable structure of Eq. (2) in a vicinity of the canard point $p_{C N}^{-} \cdot{ }^{4,18}$ Sector-type dynamics is realized when trajectories are attracted to $\left.\mathcal{Z}\right|_{\mathcal{I}_{\mathrm{can}}+\mathcal{O}(\delta)}$, where $\mathcal{I}_{\text {can }}$ is given by (A7) (we emphasize that, for sufficiently small $\delta, \mathcal{S}_{\varepsilon \delta}^{a^{-}}$and $\mathcal{S}_{\varepsilon \delta}^{r}$ intersect in a canard trajectory that provides a connection between the two manifolds; recall Subsection 1 in the Appendix). For sufficiently small $\varepsilon$ and $\delta$ and $z_{\text {in }} \in \mathcal{I}_{\text {can }}+\mathcal{O}(\delta)$, trajectories remain "trapped" and undergo SAOs ("loops"), taking $\mathcal{O}(\mu \delta \sqrt{-\varepsilon \ln \varepsilon})$ steps in the $z$-direction until they reach a point $p_{\text {out }}$ at which they can escape following the fast flow of Eq. (2). The $z$-coordinate of that point can hence be approximated by

$$
z_{\text {out }}=z_{C N}^{-}+o(1) .
$$

The number of SAOs that is observed in the corresponding trajectory is determined by the passage thereof through sectors of rotation, ${ }^{18}$ the boundaries of which are so-called "secondary" canards. Trajectories that are attracted to this region typically exhibit few SAOs of near-constant amplitude [see panel (b) of Fig. 12], where sector-type SAOs are seen in between delay-type segments. A detailed study of sector-type dynamics in Eq. (2) is part of work in progress; see again Ref. 18 for an in-depth discussion in the context of their prototypical model [Eq. (4)].

\section{REFERENCES}

${ }^{1}$ P. T. Cardin and M. A. Teixeira, "Fenichel theory for multiple time scale singular perturbation problems,” SIAM J. Appl. Dyn. Syst. 16, 1425-1452 (2017).

${ }^{2} \mathrm{R}$. Curtu and J. Rubin, "Interaction of canard and singular Hopf mechanisms in a neural model," SIAM J. Appl. Dyn. Syst. 10, 1443-1479 (2011).

${ }^{3}$ P. De Maesschalck, E. Kutafina, and N. Popović, "Three time-scales in an extended Bonhoeffer-van der Pol oscillator," J. Dyn. Differ. Equ. 26, 955-987 (2014)
${ }^{4}$ P. De Maesschalck, E. Kutafina, and N. Popović, "Sector-delayed-Hopf-type mixed-mode oscillations in a prototypical three-time-scale model," Appl. Math. Comput. 273, 337-352 (2016).

${ }^{5} \mathrm{P}$. De Maesschalck and M. Wechselberger, "Neural excitability and singular bifurcations," J. Math. Neurosci. 5, 16 (2015).

${ }^{6}$ M. Desroches, J. Guckenheimer, B. Krauskopf, C. Kuehn, H. M. Osinga, and M. Wechselberger, "Mixed-mode oscillations with multiple time scales," SIAM Rev. 54, 211-288 (2012).

${ }^{7} \mathrm{M}$. Desroches and V. Kirk, "Spike-adding in a canonical three-time-scale model: Superslow explosion and folded-saddle canards," SIAM J. Appl. Dyn. Syst. 17, 1989-2017 (2018)

${ }^{8}$ E. J. Doedel, A. R. Champneys, F. Dercole, T. F. Fairgrieve, Y. A. Kuznetsov, B. Oldeman, R. Paffenroth, B. Sandstede, X. Wang, and C. Zhang, "AUTO-07P: Continuation and bifurcation software for ordinary differential equations," 2007, see http://www.macs.hw.ac.uk/gabriel/auto07/auto.html (last accessed September 9, 2020).

${ }^{9}$ S. Doi, S. Nabetani, and S. Kumagai, "Complex nonlinear dynamics of the Hodgkin-Huxley equations induced by time scale changes," Biol. Cybern. $\mathbf{8 5}$, 51-64 (2001).

${ }^{10} \mathrm{~N}$. Fenichel, "Geometric singular perturbation theory for ordinary differential equations,” J. Differ. Equ. 31, 53-98 (1979).

${ }^{11} \mathrm{~J}$. Guckenheimer, "Singular Hopf bifurcation in systems with two slow variables," SIAM J. Appl. Dyn. Syst. 7, 1355-1377 (2008).

${ }^{12} \mathrm{~J}$. Guckenheimer and I. Lizarraga, "Shilnikov homoclinic bifurcation of mixed-mode oscillations,” SIAM J. Appl. Dyn. Syst. 14, 764-786 (2015).

${ }^{13}$ M. G. Hayes, T. J. Kaper, P. Szmolyan, and M. Wechselberger, "Geometric desingularization of degenerate singularities in the presence of fast rotation: A new proof of known results for slow passage through Hopf bifurcations," Ind. Math. 27, 1184-1203 (2016).

${ }^{14}$ A. L. Hodgkin and A. F. Huxley, "A quantitative description of membrane current and its application to conduction and excitation in nerve," J. Physiol. 117, 500-544 (1952).

${ }^{15} \mathrm{~J}$. Jalics, M. Krupa, and H. G. Rotstein, "Mixed-mode oscillations in a three timescale system of ODEs motivated by a neuronal model," Dyn. Syst. 25, 445-482 (2010).

${ }^{16} \mathrm{P}$. Kaklamanos, N. Popović, and K. U. Kristiansen, "Geometric singular perturbation analysis of the multiple-timescale Hodgkin-Huxley equations" (unpublished).

${ }^{17}$ M. T. Koper, "Bifurcations of mixed-mode oscillations in a three-variable autonomous van der Pol-Duffing model with a cross-shaped phase diagram," Physica D 80, 72-94 (1995).

${ }^{18}$ M. Krupa, N. Popović, and N. Kopell, "Mixed-mode oscillations in three timescale systems: A prototypical example," SIAM J. Appl. Dyn. Syst. 7, 361-420 (2008).

${ }^{19} \mathrm{M}$. Krupa and P. Szmolyan, "Extending geometric singular perturbation theory to nonhyperbolic points-Fold and canard points in two dimensions," SIAM J. Math. Anal. 33, 286-314 (2001).

${ }^{20}$ M. Krupa and P. Szmolyan, "Relaxation oscillation and canard explosion," J. Differ. Equ. 174, 312-368 (2001).

${ }^{21} \mathrm{M}$. Krupa and M. Wechselberger, "Local analysis near a folded saddle-node singularity,” J. Differ. Equ. 248, 2841-2888 (2010).

${ }^{22} \mathrm{C}$. Kuehn, "On decomposing mixed-mode oscillations and their return maps," Chaos 21, 033107 (2011).

${ }^{23} \mathrm{~B}$. Letson, J. E. Rubin, and T. Vo, "Analysis of interacting local oscillation mechanisms in three-timescale systems," SIAM J. Appl. Math. 77, 1020-1046 (2017).

${ }^{24} \mathrm{P}$. Nan, "Dynamical systems analysis of biophysical models with multiple timescales," Ph.D. thesis (ResearchSpace@Auckland, 2014).

${ }^{25} \mathrm{P}$. Nan, Y. Wang, V. Kirk, and J. E. Rubin, "Understanding and distinguishing three-time-scale oscillations: Case study in a coupled Morris-Lecar system," SIAM J. Appl. Dyn. Syst. 14, 1518-1557 (2015).

${ }^{26}$ A. I. Neishtadt, "Persistence of stability loss for dynamical bifurcations I," Differ. Equ. 23, 1385-1391 (1987).

${ }^{27}$ A. I. Neishtadt, "Persistence of stability loss for dynamical bifurcations II," Differ. Equ. 24, 171-176 (1988). 
${ }^{28} \mathrm{~J}$. Rubin and M. Wechselberger, "Giant squid-hidden canard: The 3D geometry of the Hodgkin-Huxley model," Biol. Cybern. 97, 5-32 (2007).

${ }^{29} \mathrm{P}$. Szmolyan and M. Wechselberger, "Canards in $\mathbb{R}^{3}$," J. Differ. Equ. 177, 419-453 (2001).

${ }^{30} \mathrm{P}$. Szmolyan and M. Wechselberger, "Relaxation oscillations in $\mathbb{R}^{3}$," J. Differ. Equ. 200, 69-104 (2004).
${ }^{31}$ T. Vo, R. Bertram, and M. Wechselberger, "Multiple geometric viewpoints of mixed mode dynamics associated with pseudo-plateau bursting," SIAM J. Appl. Dyn. Syst. 12, 789-830 (2013).

${ }^{32} \mathrm{M}$. Wechselberger, "Existence and bifurcation of canards in $\mathbb{R}^{3}$ in the case of a folded node," SIAM J. Appl. Dyn. Syst. 4, 101-139 (2005). 